\title{
GLUT5-mediated fructose utilization drives lung cancer growth by stimulating fatty acid synthesis and AMPK/mTORC1 signaling
}

\author{
Wen-Lian Chen, ${ }^{1}$ Xing Jin, ${ }^{1}$ Mingsong Wang, ${ }^{2}$ Dan Liu, ${ }^{1}$ Qin Luo, ${ }^{1}$ Hechuan Tian, ${ }^{1}$ Lili Cai, ${ }^{1}$ \\ Lifei Meng, ${ }^{2}$ Rui Bi, ${ }^{2}$ Lei Wang, ${ }^{2}$ Xiao Xie, ${ }^{2}$ Guanzhen Yu, ${ }^{1}$ Lihui Li, ${ }^{1}$ Changsheng Dong, ${ }^{1}$ Qiliang Cai, ${ }^{3}$ \\ Wei Jia, ${ }^{4}$ Wenyi Wei, ${ }^{5}$ and Lijun Jia ${ }^{1}$ \\ 'Cancer Institute, Longhua Hospital, Shanghai University of Traditional Chinese Medicine, Shanghai, China. ${ }^{2}$ Department \\ of Thoracic Cardiovascular Surgery, Xinhua Hospital, Shanghai Jiaotong University School of Medicine, Shanghai, China. \\ ${ }^{3}$ Ministry of Education and Ministry of Health Key Lab of Medical Molecular Virology, School of Basic Medical Science, \\ Fudan University, Shanghai, China. ${ }^{4}$ University of Hawaii Cancer Center, Honolulu, Hawaii, USA. ${ }^{5}$ Department of Pathology, \\ Beth Israel Deaconess Medical Center, Harvard Medical School, Boston, Massachusetts, USA.
}

Lung cancer (LC) is a leading cause of cancer-related deaths worldwide. Its rapid growth requires hyperactive catabolism of principal metabolic fuels. It is unclear whether fructose, an abundant sugar in current diets, is essential for LC. We demonstrated that, under the condition of coexistence of metabolic fuels in the body, fructose was readily used by LC cells in vivo as a glucose alternative via upregulating GLUT5, a major fructose transporter encoded by solute carrier family 2 member 5 (SLC2A5). Metabolomic profiling coupled with isotope tracing demonstrated that incorporated fructose was catabolized to fuel fatty acid synthesis and palmitoleic acid generation in particular to expedite LC growth in vivo. Both in vitro and in vivo supplement of palmitoleic acid could restore impaired LC propagation caused by SLC2A5 deletion. Furthermore, molecular mechanism investigation revealed that CLUT5-mediated fructose utilization was required to suppress AMPK and consequently activate mTORC1 activity to promote LC growth. As such, pharmacological blockade of in vivo fructose utilization using a GLUT5 inhibitor remarkably curtailed LC growth. Together, this study underscores the importance of in vivo fructose utilization mediated by CLUT5 in governing LC growth and highlights a promising strategy to treat LC by targeting CLUT5 to eliminate those fructose-addicted neoplastic cells.

Authorship note: WLC, X], MW, and DL are co-first authors.

Conflict of interest: The authors have declared that no conflict of interest exists.

Copyright: () 2020, American Society for Clinical Investigation.

Submitted: July 8, 2019

Accepted: January 10, 2020

Published: February 13, 2020.

Reference information: /CI Insight. 2020;5(3):e131596.

https://doi.org/10.1172/jci.

insight.131596.

\section{Introduction}

Lung cancer (LC) is a highly lethal malignancy and results in the largest number of cancer-related deaths worldwide $(1,2)$. The most common type of LC is non-small cell lung cancer ( $85 \%$ of all LC), which is generally subcategorized into adenocarcinoma (ADC, $40 \%$ of all LC), squamous cell carcinoma (SCC, $25 \%-30 \%$ of all LC), and large cell carcinoma (10\%-15\% of all LC) (3). Genetic aberrations involved in LC pathogenesis have been thoroughly studied during past decades, leading to the identification of a common set of oncogenes, including EGFR, KRAS, BRAF, ALK, RET, and ROS1 (3, 4). Novel targeted drugs were developed consequently to improve LC therapy (1). Recently, immune evasion in LC is particularly focused on, and checkpoint inhibitor therapies are designed for patients with LC, especially for those without actionable driver mutations (5). Despite these achievements, the 5 -year survival rate in the LC population is still less than $20 \%(1,2)$. Therefore, there is an increasing need to comprehensively understand the mechanisms involved in LC initiation and progression.

Recent studies demonstrate that metabolic reprogramming is a core hallmark of cancer and contributes to cancer initiation and progression (6). Exploiting metabolic targets would yield new therapeutic opportunities for cancer patients $(6,7)$. For LC, its global metabolism is remarkably altered, and many metabolic pathways are dysregulated to promote LC progression (8-10). Among these pathways, glucose metabolism is studied intensively because this pathway is widely used by cancer cells to provide building blocks and to acquire therapeutic resistance (11-13). LC exhibits a feature of hyperactive glucose metabolism as verified by metabolomic studies and clinical PET-CT examinations $(8,14)$. Glucose transporters, including GLUT1 and GLUT3, are found to be upregulated in LC cells to support their increased glucose uptake $(15,16)$. 
Elevated activity of glucose metabolism is closely associated with inferior survival of patients with LC (15, 17). Inhibiting this metabolic pathway impedes LC growth and progression (18). Collectively, highly active glucose metabolism is critical to sustain LC malignancy.

However, substantial consumption of glucose by LC cells to retain the glucose metabolism activity would inevitably cause glucose insufficiency in the tumor microenvironment, which is adverse for LC cells $(8,19)$. Therefore, alternative metabolic fuels are required for these malignant cells. Fructose, which is plentiful in current diets, is the second most abundant blood sugar in humans (20-24). Dietary fructose is predominantly absorbed from intestinal lumen via the hexose transporters GLUT2 and GLUT5 in an insulin-independent manner $(20,25)$. Under physiological conditions, $70 \%$ of portal fructose is removed by the liver, and the remaining $30 \%$ is metabolized by other tissues (26). Fructose incorporated in hepatic cells enters the glycolytic pathway, completely bypassing 2 of 3 highly regulated rate-limiting steps, and the glycolytic intermediates are used mainly for lipogenesis $(26,27)$. There are 3 enzymes involved in fructose metabolism, including ketohexokinase, aldolase B, and triokinase (28).

GLUT5, one of the major fructose transporters, is upregulated in LC, and lung ADC cell lines cultured in dishes are able to use fructose as an alternative metabolic fuel (29). However, a fundamental question is still to be answered. Under the condition of coexistence of other alternative metabolic fuels in the body, it is unknown whether fructose is used by LC cells in vivo via GLUT5 to support cellular metabolic activity and to promote LC growth. In this study, we will focus on this question and deeply investigate the activity, underlying tumor-promoting mechanism, and therapeutic potential of in vivo fructose utilization mediated by GLUT5 in LC.

\section{Results}

In vivo fructose utilization is activated in LC tissues of patients. To assess in vivo fructose metabolism activity in LC, we enrolled 2 LC patient cohorts with ADC $(n=22)$ or SCC $(n=13)$ (Figure 1A) and conducted a metabolomic investigation using paired tumorous and adjacent normal lung tissues from these patients. As compared with adjacent normal tissues, LC tissues contained increased glycolytic intermediates, including glucose-6-phosphate and fructose-6-phospate, but reduced glucose (Supplemental Figure 1, A and B; supplemental material available online with this article; https://doi.org/10.1172/jci.insight.131596DS1). This indicated that glycolytic activity was strongly activated in LC tissues, thus causing glucose insufficiency in the tumor microenvironment. Notably, the levels of fructose, the ratio of fructose and glucose, and fructose-derived metabolites, including fructose-1-phosphate and dihydroxyacetone phosphate (DHAP), were raised in LC tissues (Figure 1, B and C, and Supplemental Figure 1C). Therefore, we hypothesized that in vivo LC cells of patients could switch to use circulating fructose when glucose was scarce. To support this hypothesis, we collected fresh and paired tumorous and adjacent normal lung tissues from operative patients to perform an ex vivo fructose uptake assay. When glucose was sufficient in the medium (6 mM), both tumor and adjacent normal tissues exhibited low fructose uptake (Figure 1D). However, when glucose level was low or deprived, tumor tissues significantly upregulated their fructose consumption rate, whereas nontumor tissues did not reveal this metabolic shift (Figure 1D). These findings suggested that fructose was an alternative carbon source and its metabolism was enhanced in LC tissues under glucose-limiting conditions, whereas matched adjacent normal lung tissues did not show this fructose-addicted feature.

To further determine the metabolic diversion to fructose utilization of LC cells, we enrolled several representative LC cell lines and measured 3 parameters in vitro, including fructose uptake, fructose-induced cell proliferation, and fructose-induced suppression of cell apoptosis. Five LC cell lines with different genetic backgrounds were used, including 2 lung ADC cell lines (A549 and EKVX), 2 lung SCC cell lines (NCI-H226 and SK-MES-1), and 1 large cell lung carcinoma line (Hop-92). All these cell lines exhibited elevated fructose uptake and increased fructose-induced cell proliferation under conditions of glucose deficiency ( 0 or $0.75 \mathrm{mM}$ ) compared with glucose sufficiency $(6 \mathrm{mM})$ (Supplemental Figure $1 \mathrm{D}$ and Supplemental Figure 2A). Finally, all enrolled LC cells could harness fructose to reduce cell apoptosis caused by glucose deprivation (Supplemental Figure 2, B-E). Taken together, these results demonstrated that LC cells in vitro could switch to use fructose to sustain growth and survival under glucose-limited conditions.

GLUT5 mediates in vivo fructose utilization to support LC growth. Fructose is imported into cells largely via 2 major fructose transporters, GLUT2 and GLUT5, which are encoded by solute carrier family 2 members 2 and 5 (SLC2A2 and SLC2A5), respectively (20, 30, 31). Analysis of The Cancer Genome Atlas (TCGA) RNA-Seq data and Gene Expression Omnibus microarray data revealed that $S L C 2 A 2$ expression was not significantly altered in LC tissues of patients with ADC or SCC (Figure 2, A and B), whereas the expression of 
A

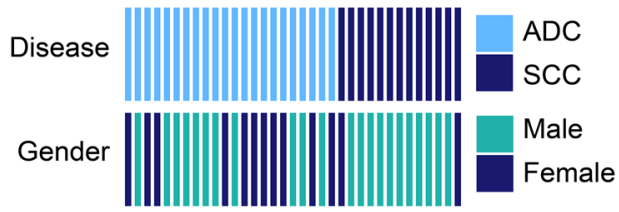

Age, years

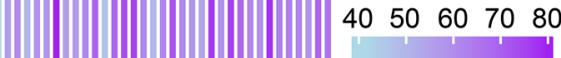

Weight, kg

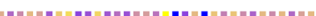

405060708090

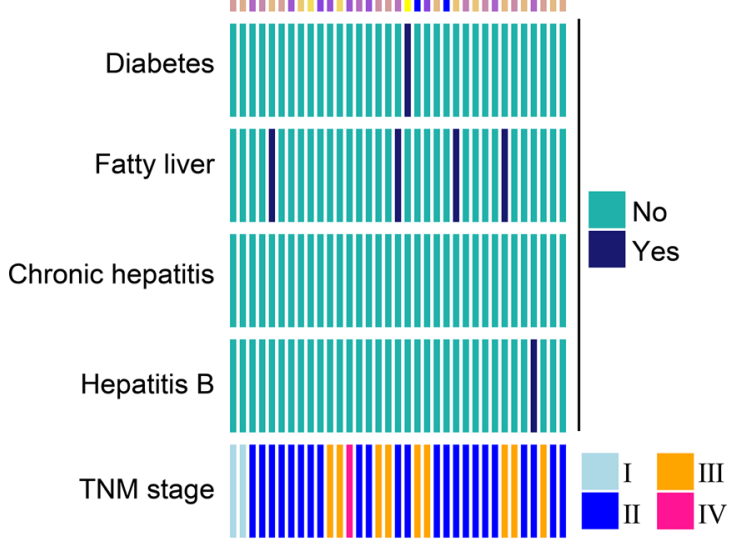

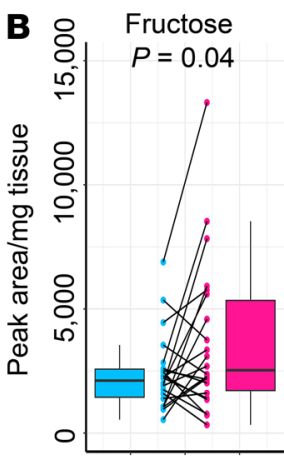

C

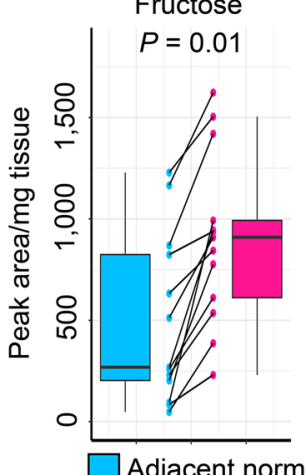

Adjacent normal tissues
Fructose-1-P
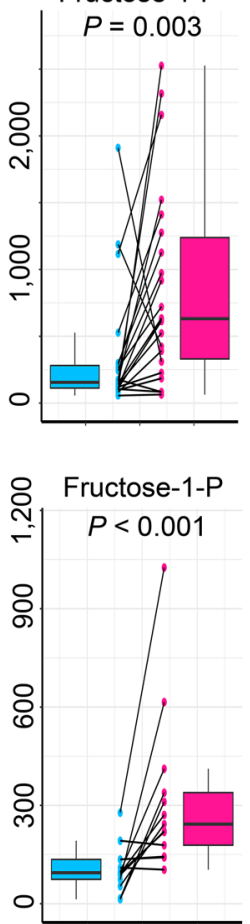

DHAP
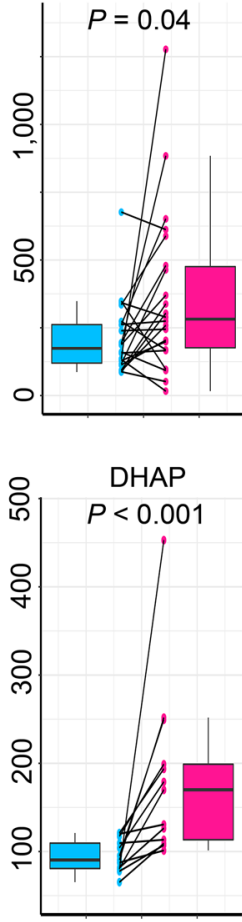

Tumor tissues

D

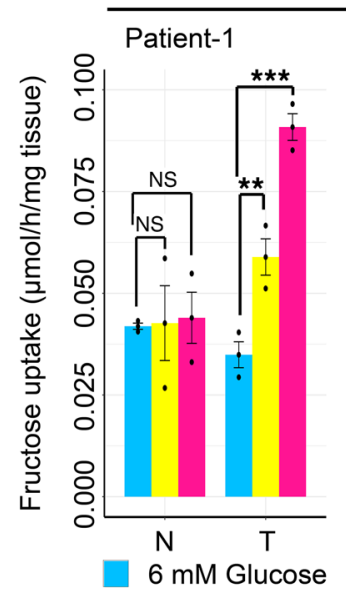

Adenocarcinoma lung tissues

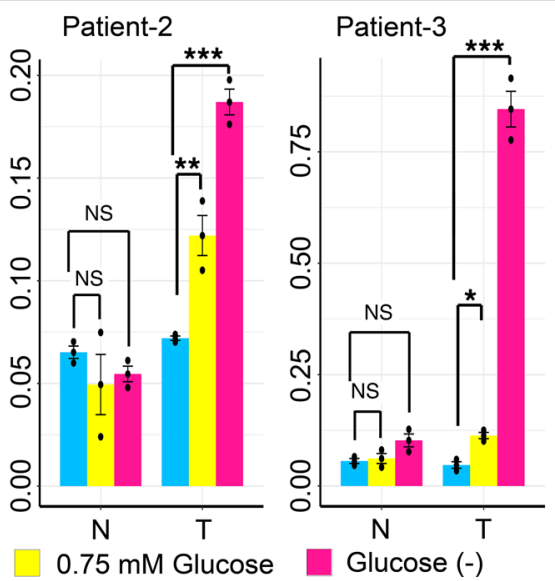

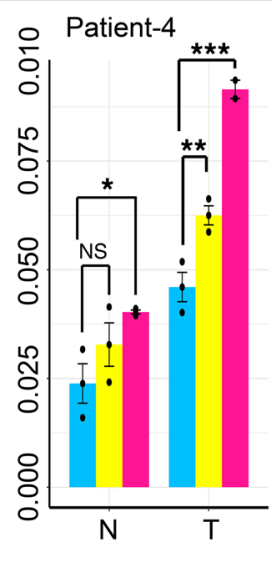

Squamous cell carcinoma lung tissues

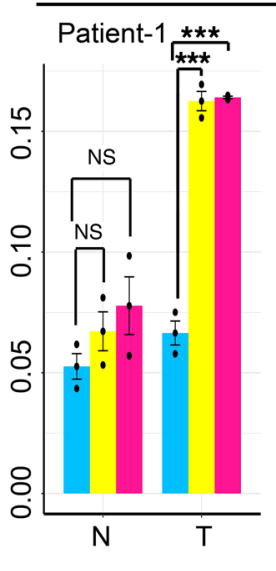

Figure 1. Activity of fructose utilization in LC tissues of patients. (A) Visualization of clinical parameters of enrolled patients in the study for metabolomic survey. ADC, $n=22$; SCC, $n=13$. TNM, extent of the primary tumor, involvement of lymph nodes, and distant metastases. (B and C) Concentrations of fructose and fructose-derived metabolites between paired adjacent normal lung tissues and tumor tissues from patients with lung ADC ( $n=22$ ) (B) or SCC $(n=13)(C)$. The midline represents the median of the data, with the upper and lower limits of the box being the third and first quartiles. Additionally, the whiskers of the box plot extend up to 1.5 times the interquartile range from the top or bottom of the box. $P$ values were computed using 2 -tailed Wilcoxon rank-sum test. Fructose-1-P, fructose-1-phosphate; DHAP, dihydroxyacetone phosphate. (D) Ex vivo study of fructose uptake between paired adjacent normal lung tissues $(\mathrm{N})$ and tumor lung tissues $(\mathrm{T})$ from patients with lung ADC or SCC. Tissues were cultured in complete medium containing $1.5 \mathrm{mM}$ fructose and different levels of glucose for 48 hours. Statistical analysis was conducted using 1-way ANOVA test. After conducting a homogeneity of variance test to confirm equal variances among subgroups, $P$ values were obtained from post hoc test using least significant difference (LSD) method. Cumulative data are shown; $n=3$. Data shown as mean $\pm \mathrm{SEM} ; n=3$. ${ }^{*} P<0.05 ;{ }^{* *} P<0.01 ;{ }^{* *} P<0.001,2$-tailed Student's $t$ test.

SLC2A5 was markedly upregulated in the neoplastic lung tissues of TCGA patients and our patient cohorts (Figure 2, C and D). Correspondingly, the protein product of SLC2A5, GLUT5, was elevated in LC tissues of our patient cohorts (Figure 2, E and F; see complete unedited blots in the supplemental material.). Furthermore, we enrolled 2 independent LC patient cohorts with ADC or SCC to conduct an immunohistochemistry (IHC) assay. The results verified that GLUT5 was upregulated in LC tissues of patients (Figure 3, A and B). 
Together, these findings demonstrated that GLUT5, but not GLUT2, was aberrantly upregulated in LC tissues of patients. Notably, high GLUT5 expression in LC tissues measured by IHC staining predicted unfavorable overall survival of patients (Figure 3, C and D).

Subsequently, we determined whether GLUT5 mediated in vivo fructose uptake of lung tumors. A549 and EKVX cells with distinct genetic aberrations (32) were enrolled to abrogate SLC2A5 using 2 distinct guide RNAs, respectively (Figure 4A). In vitro, $S L C 2 A 5$ deletion markedly suppressed fructose uptake and fructose-induced cell proliferation yet negligibly influenced glucose uptake and glucose-induced cell proliferation (Figure 4, B and C, and Supplemental Figure 3). In vivo, SLC2A5 abrogation reduced ${ }^{13} \mathrm{C}$-fructose level and restrained LC xenograft growth (Figure 5, A-F, and Supplemental Figure 4, A-E). Consistently, SLC2A5 deletion downregulated the expression of 2 tumor growth markers, proliferating cell nuclear antigen (PCNA) and Ki-67, as well as a cell cycle progression marker, cyclinD1, in LC xenografts (Figure 5, G-I). In addition, SLC2A5 abrogation elevated the expression of a cell apoptosis marker, cleaved caspase-3 (Supplemental Figure 4F), and repressed an angiogenesis marker, CD31, in A549 xenografts (Supplemental Figure 4G).

Because high expression of GLUT5 was closely linked to poor patient survival, we assumed that increased GLUT5 expression would exacerbate LC malignancy and promote disease progression. To test this assumption, we ectopically enforced GLUT5 expression in 3 LC cell lines, including A549, Hop-92, and SK-MES-1 (Supplemental Figure 5A). Compared with the control cells, LC cells with ectopic GLUT5 displayed significant increases in fructose uptake and fructose-induced cell proliferation in vitro under conditions of no or low glucose (Supplemental Figure 5, B-D). Of note, the in vivo study exhibited that enforced expression of GLUT5 in A549 cells significantly increased ${ }^{13} \mathrm{C}$-fructose level in tumor xenografts, accelerated subcutaneous tumor growth, and upregulated PCNA expression (Figure 6, A-E). In conclusion, enhanced fructose utilization mediated by GLUT5 is capable of expediting the neoplastic growth of LC cells in vitro and in vivo.

In vivo fructose utilization mediated by GLUT5 fuels fatty acid synthesis and palmitoleic acid generation in lung tumors. To elucidate the underlying metabolic mechanism of fructose-induced LC growth, we surveyed the in vivo influence of GLUT5-mediated fructose utilization on global metabolism of LC xenografts. SLC2A5 abrogation in A549 xenografts reduced fructose, fructose-1-phosphate, fatty acid precursors (citric acid and acetyl-CoA), and fatty acids, while ectopic GLUT5 expression in A549 tumors raised these metabolites (Figure 7, A and B). Thus, we concluded that in vivo fructose utilization mediated by GLUT5 was required for maintaining the homeostasis of the endogenous fatty acid pool in lung tumors.

To elucidate whether fructose was directly involved in fatty acid synthesis, we conducted in vivo metabolic flux assays. We intravenously infused ${ }^{13} \mathrm{C}$-fructose into mice and traced the downstream metabolites in A549 xenografts. We observed that ${ }^{13} \mathrm{C}$-pyruvate, ${ }^{13} \mathrm{C}$-acetyl-CoA, and ${ }^{13} \mathrm{C}$-palmitoleic acid were reduced in A549 tumors with SLC2A5 abrogation, whereas these metabolites were upregulated in A549 tumors with enforced GLUT5 expression (Figure 7, C and D). Notably, ${ }^{13} \mathrm{C}$-lactate was not disturbed by SLC2A 5 deletion or GLUT5 overexpression in A549 tumor xenografts (Supplemental Figure 6, A and B). Furthermore, we implemented an ex vivo study using LC tissue slices with high GLUT5 and paired adjacent normal tissue slices with low GLUT5 from 5 patients with lung ADC. These tissues were cultured in medium containing ${ }^{13} \mathrm{C}$-fructose. We observed that ${ }^{13} \mathrm{C}$-fructose was dramatically accumulated, and the production of ${ }^{13} \mathrm{C}$-fructose-derived metabolites, including ${ }^{13} \mathrm{C}$-pyruvate, ${ }^{13} \mathrm{C}$-acetyl-CoA , and ${ }^{13} \mathrm{C}$-palmitoleic acid, was increased in the LC slices (Figure 7E). In addition, 3 fatty acids were dramatically upregulated $(P<0.05)$, while 4 fatty acids had an increased trend $(P<0.1)$ in ADC tissues of patients (Figure $7 \mathrm{~F})$. Moreover, 4 fatty acids had an increased trend $(P<0.1)$ in SCC tissues of patients (Supplemental Figure 6C). Collectively, these findings demonstrated that absorbed fructose was used to synthesize fatty acids, including palmitoleic acid, in lung tumors.

Next, we ascertained whether fatty acid synthesis fueled by fructose was essential to LC growth. We suppressed fatty acid synthesis by deleting the key enzyme fatty acid synthase (FASN) or by using an FASN inhibitor, C75 (33). When using fructose as the carbon source, both FASN knockout and C75 treatment significantly repressed LC cell growth (Figure 8, A-C, and Supplemental Figure 6D). Moreover, when using fructose as the carbon source, supplement of a fructose-derived fatty acid, palmitoleic acid, could significantly restore the impaired growth of LC cells with $S L C 2 A 5$ deletion (Figure $8 \mathrm{D}$ and Supplemental Figure $6 \mathrm{E}$ ). Consistently, oral administration of palmitoleic acid to mice could restore the growth of A549 xenografts with $S L C 2 A 5$ ablation (Figure 8, E and F). Of note, when 
A
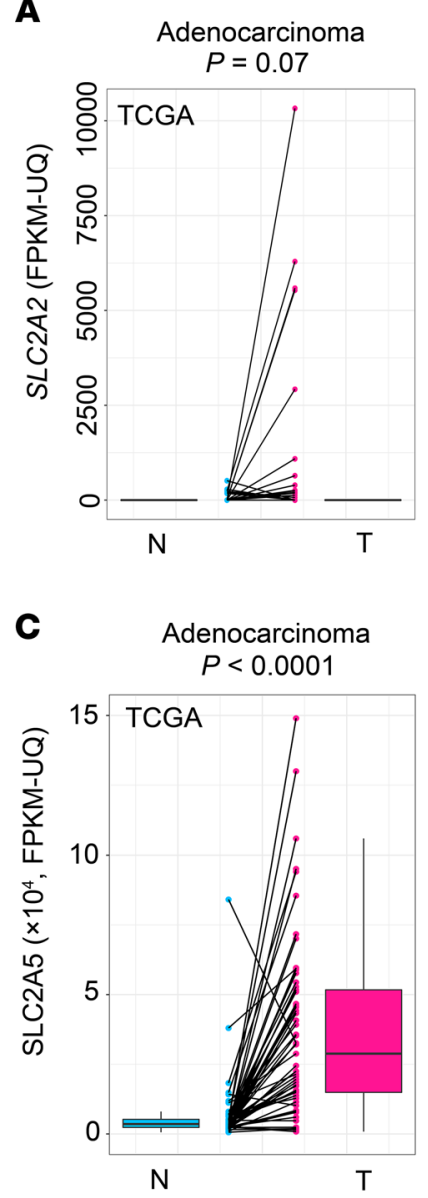

$\mathbf{E}$

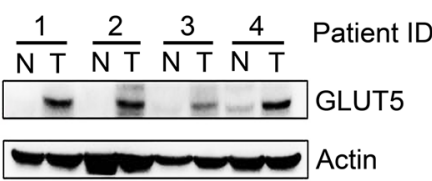

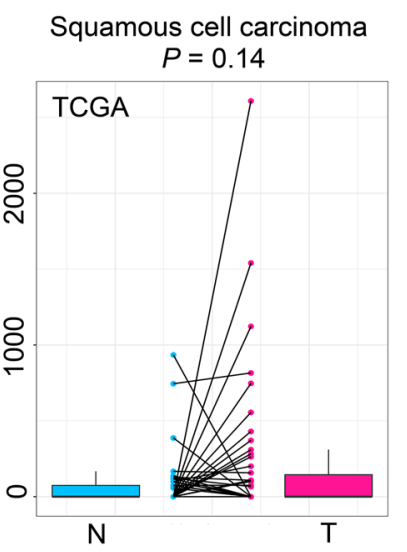
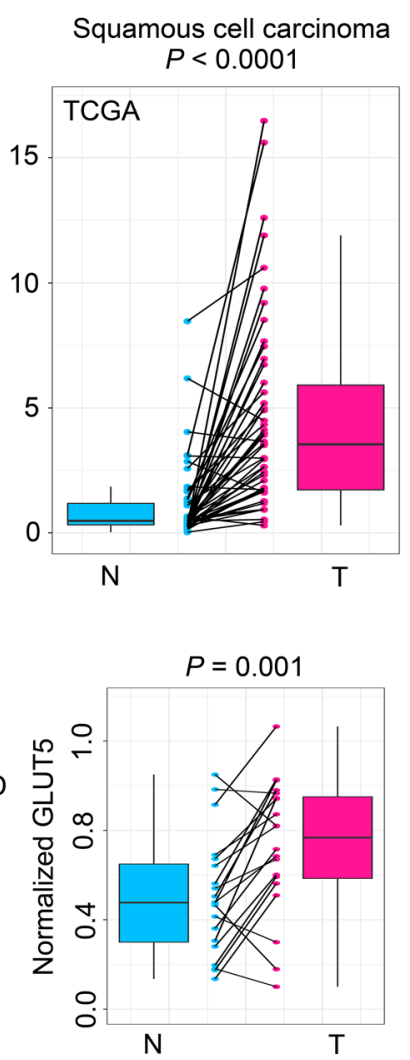

B
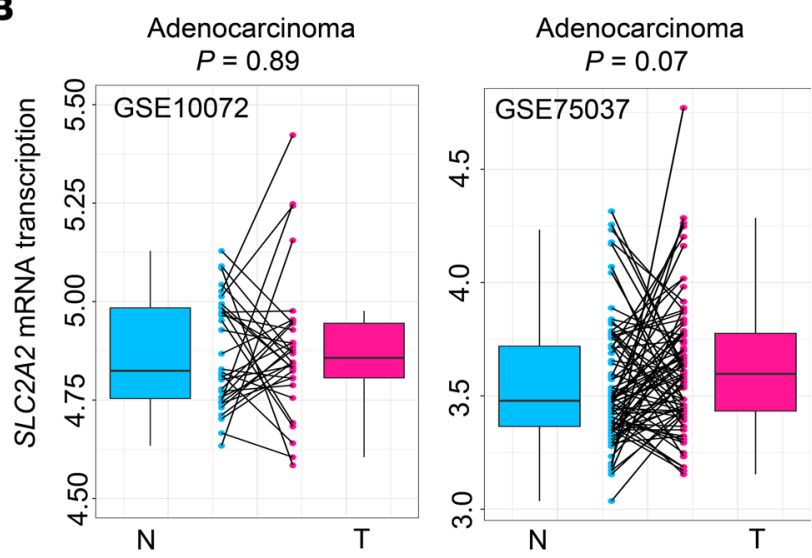

D
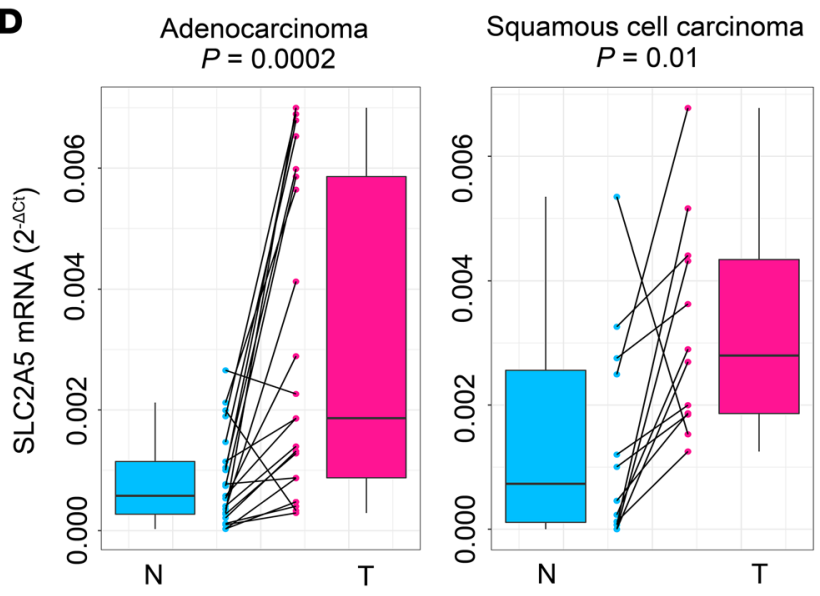

$\mathbf{F}$

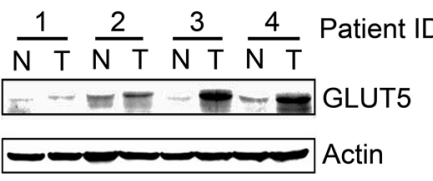

Figure 2. GLUT5 not GLUT2 is upregulated in LC tissues of patients. (A and B) SLC2A2 expression between paired adjacent normal (N) and tumorous (T) lung tissues of patients with LC. Data were obtained from public databases, including 2 TCGA RNA-Seq data sets $(n=57$ for patients with ADC and $n=$ 50 for patients with SCC) (A) and 2 microarray data sets of GSE10072 $(n=32)$ and GSE75037 $(n=83)$ (B). (C) TCGA data sets showing SLC2A5 expression between paired adjacent normal and tumorous lung tissues of patients with lung ADC $(n=57)$ or SCC $(n=50)$. (D) SLC2A5 expression between paired adjacent normal and tumorous lung tissues from patients with lung ADC $(n=21)$ or SCC $(n=12)$ from our hospital. (E and F) Representative Western blot images and statistical analysis of GLUT5 expression between paired adjacent normal and tumorous tissues from patients with lung ADC ( $n=20)(\mathbf{E})$ or SCC $(n=12)(\mathbf{F})$. Actin was used to normalize GLUT5 expression. For box plots, the midline represents the median of the data, with the upper and lower limits of the box being the third and first quartiles. Additionally, the whiskers of the box plot extend up to 1.5 times the interquartile range from the top or bottom of the box. $P$ values were calculated using 2-tailed Wilcoxon's rank-sum test.

endogenous generation of palmitoleic acid from fructose was not disturbed, exogenous supplement of this fatty acid did not alter LC growth in vitro and in vivo (Figure 8, D-F, and Supplemental Figure 6E). Together, these results demonstrated that fructose-derived fatty acids, including palmitoleic acid, were vital for in vivo LC propagation.

In vivo fructose utilization mediated by GLUT5 modulates AMPK/mTORC1 signaling in lung tumors. To comprehensively understand the molecular mechanism underlying fructose utilization-induced LC 
A
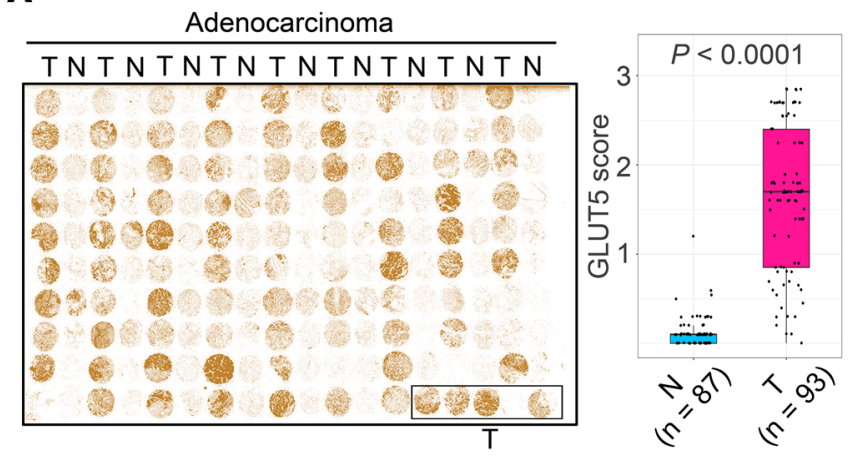

C

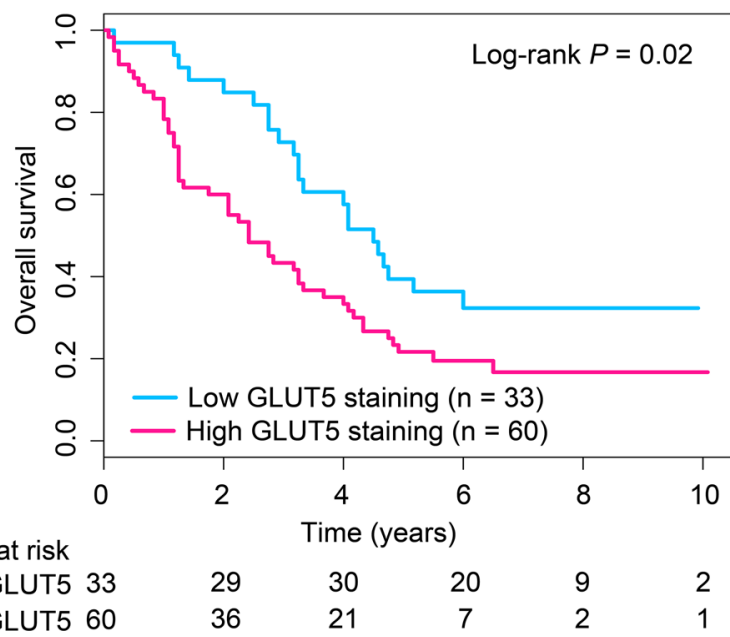

B

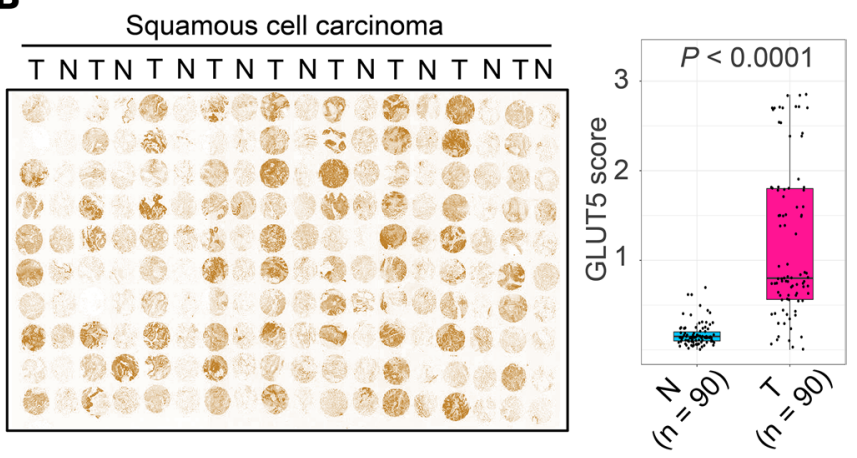

D

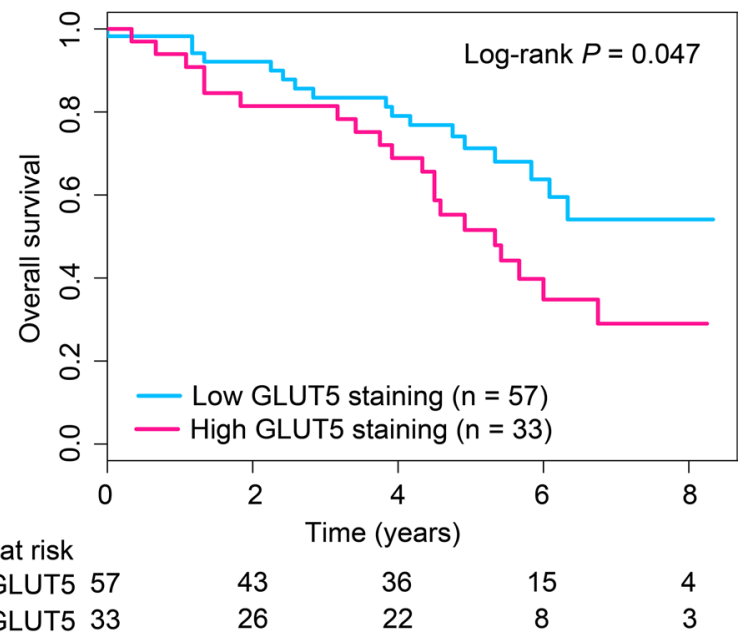

Figure 3. Increased GLUT5 expression in LC tissues is validated in $\mathbf{2}$ independent patient cohorts. (A and B) IHC staining of lung tissue microarrays using CLUT5 antibody for patients with lung ADC (A) or SCC (B). N, paired adjacent normal lung tissues; T, tumorous lung tissues. The midline represents the median of the data, with the upper and lower limits of the box being the third and first quartiles. Additionally, the whiskers of the box plot extend up to 1.5 times the interquartile range from the top or bottom of the box. $P$ values were calculated using 2-tailed Wilcoxon's rank-sum test. (C and $\mathbf{D})$ Overall survival curves of patients with lung ADC (C) or lung SCC (D) stratified by low and high GLUT5. GLUT5 expression in LC tissues was measured by IHC staining. $P$ values were computed using log-rank test.

growth, we selected an A549 tumor xenograft with $S L C 2 A 5$ deletion as well as a control A549 tumor xenograft to perform a phospho-proteomics-based study using a phospho-antibody microarray, to detect protein phosphorylation events at specific sites and to identify those downstream substrates/effectors that modulate LC growth. When compared with the control tumor, the A549 tumor with SLC2A5 deletion contained 82 proteins whose phosphorylation levels were increased more than $50 \%$ and 46 proteins whose phosphorylation levels were decreased more than 50\% (Supplemental Figure 7A). Among these proteins with altered phosphorylation levels in the A549 tumor with $S L C 2 A 5$ deletion, the top protein was CoA carboxylase 1 (ACC1), with a phosphorylation level increased more than $838 \%$ at Ser79. ACC1 is a well-known downstream target of AMPK (34). Correspondingly, AMPK was remarkably activated as demonstrated by its phosphorylation at Thr172 with a fold change of 1.95 (Supplemental Figure 7A). Therefore, we focused on the AMPK pathway in the A549 tumor with SLC2A5 deletion.

Subsequently, we analyzed the well-established AMPK downstream targets (35) in the A549 tumor with $S L C 2 A 5$ deletion using the phospho-antibody microarray data. Among those altered downstream targets (Supplemental Figure 7B), mTORC1 activity was closely linked to LC cell growth (36). Phosphoantibody microarray data showed that phosphorylation of the 2 best characterized substrates of mTORC1, 4E-BP1 and P70S6K (37), was remarkably suppressed (Figure 9A and Supplemental Figure 7B). We then validated this result using the Western blot approach. The results demonstrated that, in A549 tumors with SLC2A5 abrogation, phosphorylation of AMPK at Thr172 and ACC1 at Ser79 was enhanced, whereas phosphorylation of $4 \mathrm{E}-\mathrm{BP} 1$ at $\mathrm{Thr} 37$ and Thr46 was attenuated, indicating that blockage of fructose utilization by SLC2A5 deletion activated AMPK and then inhibited mTORC1 activity (Figure 9B). 
A

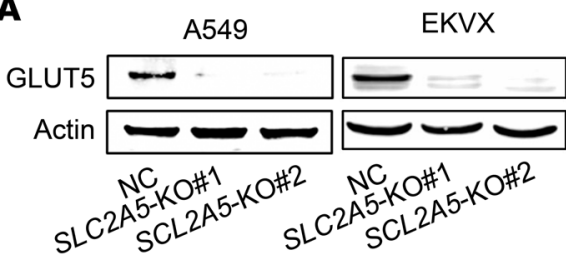

B

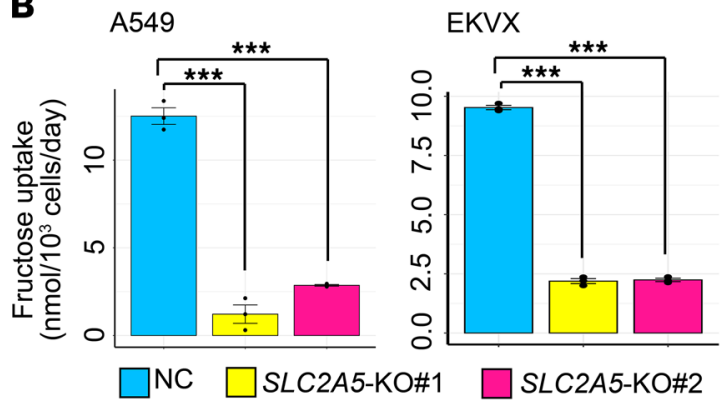

C

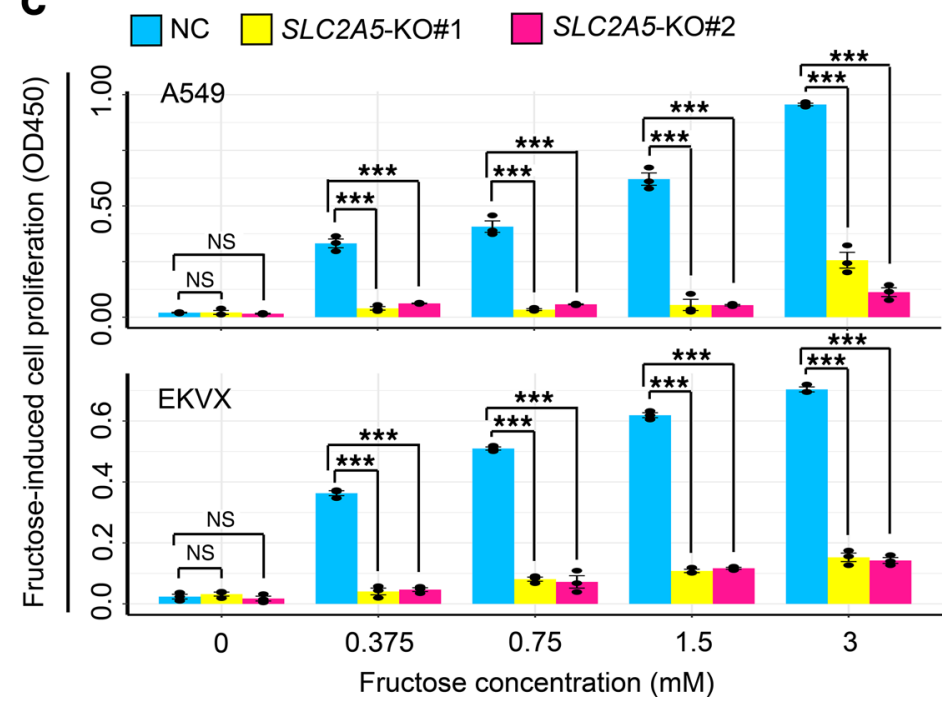

Figure 4. Abrogation of SLC2A5 impairs fructose uptake and fructose-induced cell proliferation of LC cells in vitro. (A) Western blot showing CRISPR/ Cas9-mediated deletion of SLC2A5 in A549 and EKVX cells. (B) Fructose uptake by A549 and EKVX cells with or without SLC2A5 ablation in vitro. Statistical analysis was conducted using 1-way ANOVA test. After carrying out a homogeneity of variance test to confirm equal variance among subgroups, $P$ values were acquired from post hoc test using LSD method. Cumulative data are shown; $n=3$. (C) Fructose-induced cell proliferation of A549 and EKVX cells with or without SLC2A5 abrogation in vitro. Cells were cultured in fructose medium for 72 hours. Statistical analysis was conducted using 1-way ANOVA test. After performing a homogeneity of variance test to verify equal variance among subgroups, $P$ values were gained from post hoc test using LSD method. Cumulative data are shown; $n=3$. NC, nontarget control; KO, knockout. Data shown as mean \pm SEM. ${ }^{* * *} P<0.001,2$-tailed Student's $t$ test.

In agreement with this finding, RNA-Seq data revealed that the well-established downstream target genes of mTORC1 (38) were significantly downregulated in A549 tumors with SLC2A5 ablation (Figure 9C). In addition, another well-known downstream target, p53 of AMPK, was upregulated in A549 tumors with $S L C 2 A 5$ deletion (Supplemental Figure 7C). Notably, Bax, a substrate of p53, was increased in A549 tumors with $S L C 2 A 5$ deletion (Supplemental Figure 7C).

To further confirm that GLUT5-mediated fructose utilization could affect the AMPK/mTORC1 signaling pathway, we conducted the following in vitro experiments. Control A549 cells and A549 cells with $S L C 2 A 5$ knockout were starved in sugar-free medium for 2 hours to stimulate AMPK and suppress mTORC1 activity. Then fructose and glucose were added into the media, respectively, at a final concentration of 6 $\mathrm{mM}$ to treat cells for 1 hour. Under the fructose condition, control A549 cells could use fructose to inhibit AMPK activity and upregulate mTORC1 activity, whereas A549 cells with $S L C 2 A 5$ deletion did not exhibit this signal alteration because of loss of fructose utilization capability (Figure 9, D and E). Of note, SLC2A5 deletion did not influence A549 cells to use glucose to inhibit AMPK activity and to activate mTORC1 activity consequently (Figure 9, D and E). Furthermore, to verify whether activated AMPK impeded LC cell growth, we used an AMPK agonist, AICAR, to aberrantly stimulate AMPK and to reduce mTORC1 activity consequently in A549 cells (Figure 9F). We observed that A549 cell proliferation was significantly restrained by AICAR treatment (Figure 9G). In conclusion, GLUT5-mediated fructose utilization in LC cells was required to suppress AMPK and to activate MTORC1 activity, thus promoting LC cell growth.

In vivo pharmacological blockage of fructose utilization in LC reveals therapeutic potential. Because fructose utilization mediated by GLUT5 was essential for LC growth, we asked whether pharmacologically blocking fructose utilization would restrain LC growth. We selected 2,5-anhydro-D-mannitol (2,5-AM), a specific inhibitor of GLUT5 (39), to test its efficacy on LC. We observed that 2,5-AM treatment inhibited fructose-induced cell proliferation of A549 and EKVX in a dose-dependent manner under no- or low-glucose conditions, whereas this reagent showed a negligible effect on glucose-induced LC cell propagation (Supplemental Figure 8A). Moreover, 2,5-AM repressed fructose-induced colony growth of LC cells (Supplemental Figure 8B). These results demonstrated that 2,5-AM was effective and specific to block fructose utilization of LC cells in vitro.

Subsequently, we asked whether in vivo 2,5-AM administration could suppress fructose utilization of lung tumors and generate a therapeutic effect. We found that intraperitoneal infusion of 2,5-AM 
A

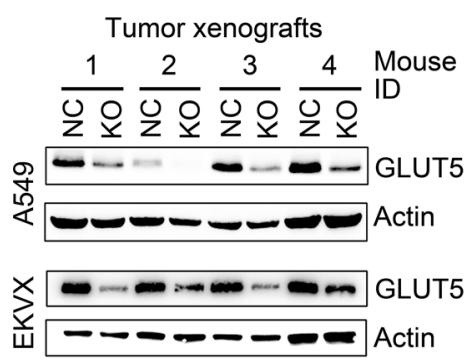

D

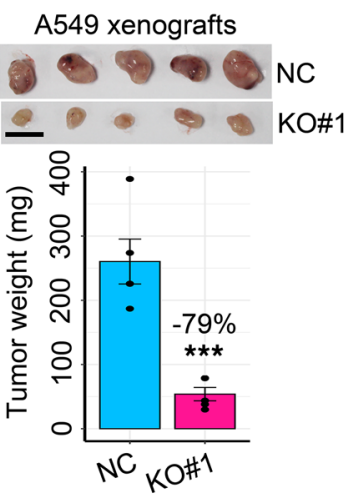

G

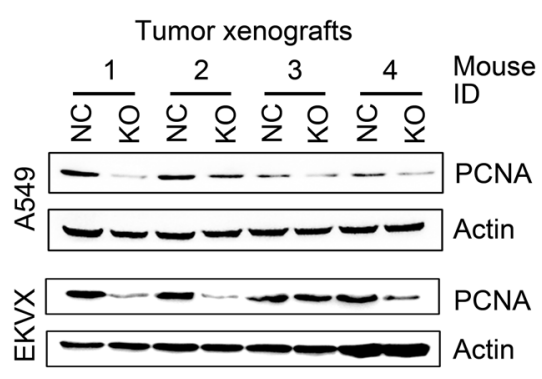

B

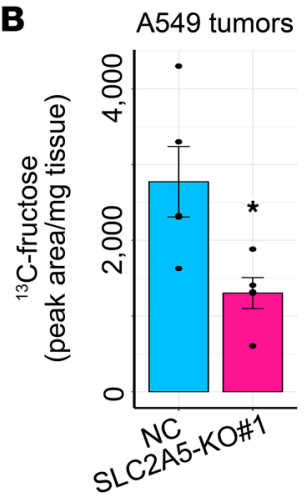

E

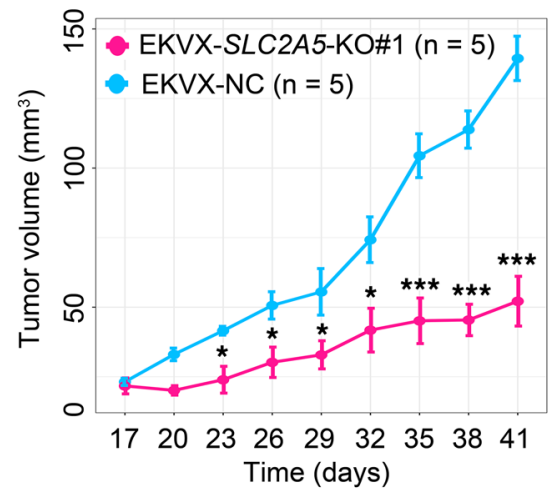

H
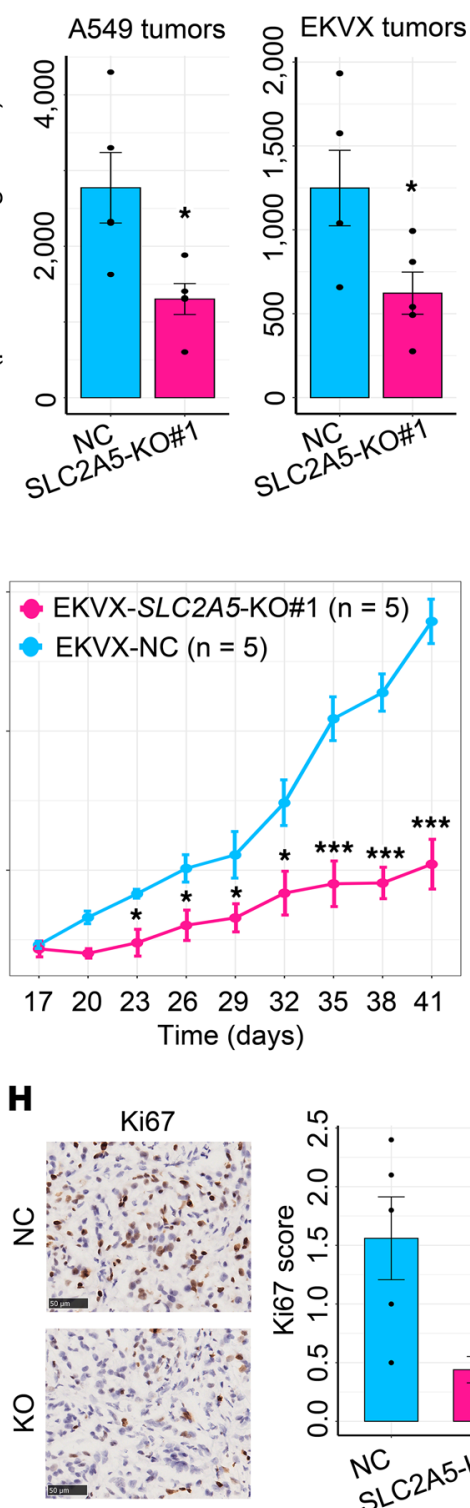

SLC2

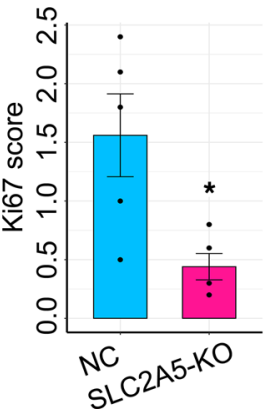

Time (days)

$\mathbf{F}$

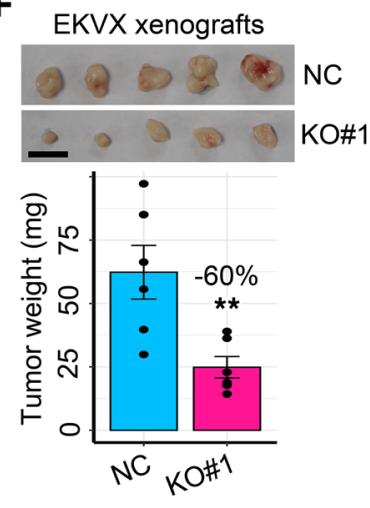

I

C
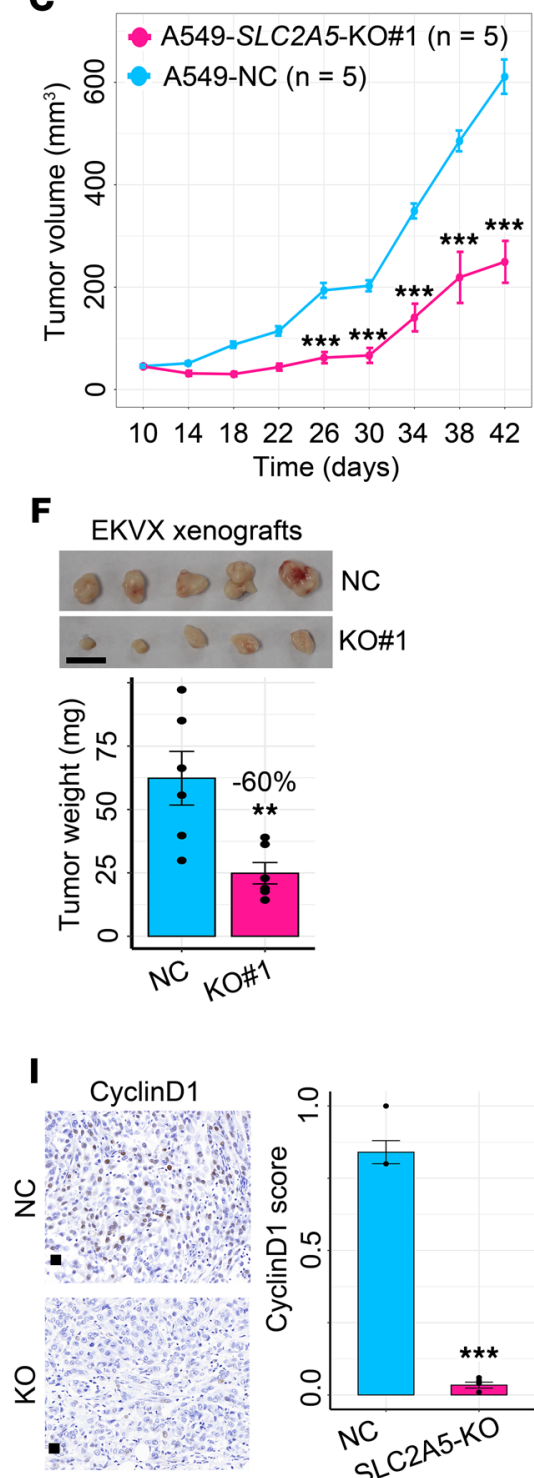

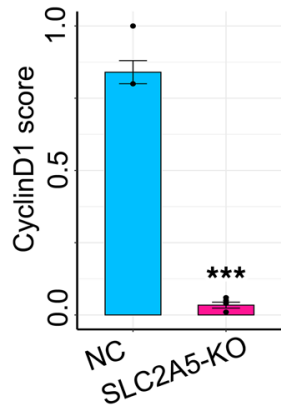

Figure 5. Blockade of in vivo fructose utilization via deleting SLC2A5 hinders the neoplastic growth of LC xenografts. (A) GLUT5 expression in LC xenografts with or without SLC2A5 abrogation. (B) ${ }^{13}$ C-fructose concentration in A549 ( $n=5$ tumors for each group) and EKVX ( $n=5$ tumors for each group) xenografts with or without SLC2A5 deletion. (C) Subcutaneous tumor growth of A549 cells with or without SLC2A5 deletion in nude mice. (D) Xenograft tumor images and tumor weight of A549 cells with or without SLC2A5 ablation ( $n=5$ tumors for each group). Scale bar: $1 \mathrm{~cm}$. (E) Subcutaneous tumor growth of EKVX cells with or without GLUT5 ablation in nude mice ( $n=5$ tumors for each group). (F) Tumor images and tumor weight of EKVX cells with or without SLC2A5 abrogation ( $n=5$ tumors for each group). Scale bar: $1 \mathrm{~cm}$. (G) Representative Western blot images displaying PCNA expression in A549 and EKVX tumor xenografts with or without SLC2A5 ablation. (H) Representative IHC images and quantitative bar plot showing Ki-67 expression in A549 tumor xenografts with or without SLC2A5 deletion ( $n=5$ tumors for each group). Scale bar: $50 \mu \mathrm{m}$. (I) Representative IHC images and quantitative bar plot showing cyclinD1 expression in A549 tumor xenografts with or without SLC2A5 deletion ( $n=5$ tumors for each group). Scale bar: $20 \mu \mathrm{m}$. Data shown as mean $\pm \mathrm{SEM}$. ${ }^{*} P<0.05 ;{ }^{* *} P<0.01 ;{ }^{* *} P<0.001$, 2-tailed Student's $t$ test.

repressed fructose input and fructose-1-phosphate generation in LC xenografts (Figure 10, A and B). Consequently, LC xenograft growth was inhibited (Figure 10, C and D), indicating that in vivo 2,5AM administration exhibited therapeutic efficacy. Mechanistically, in vivo 2,5-AM administration reduced fatty acid synthesis of LC xenografts (Figure 10, E and F). Notably, there were no obvious side effects for in vivo 2,5-AM administration on mice, as shown by the undisturbed body weight and blood concentrations of alanine transaminase and aspartate transaminase (Supplemental Figure 8, C-F). Collectively, this study delineated that a "GLUT5-fructose utilization-fatty acid synthesis-AMPK/ mTORC1" axis played a crucial role in governing LC growth, while pharmacological blockade of fructose utilization using a GLUT5 inhibitor showed promising therapeutic potential (Figure 10G). 
A

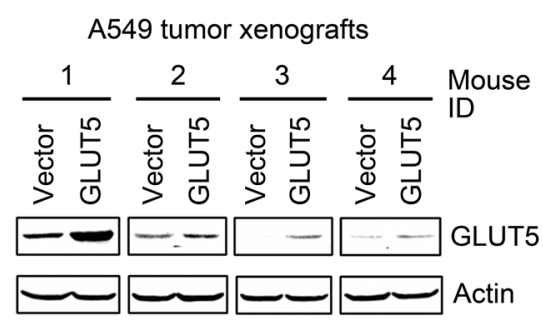

C

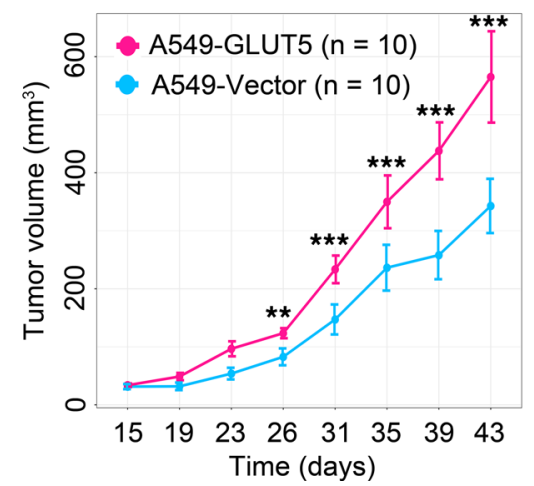

B

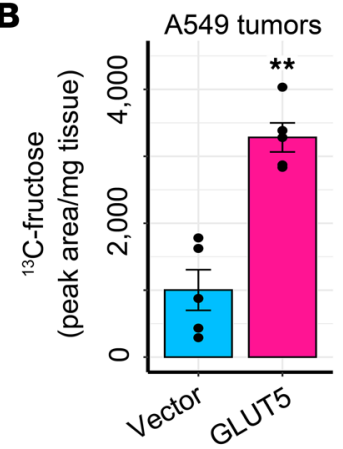

D

A549 tumors

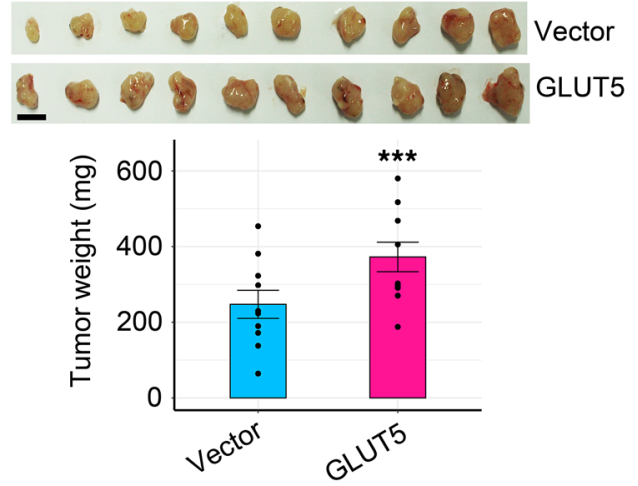

E

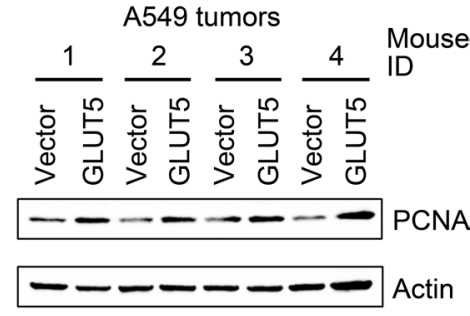

Figure 6. Enhanced fructose utilization via enforcing GLUT5 expression exacerbates the malignancy of LC xenografts. (A) Western blot showing GLUT5 expression in A549 tumor xenografts with or without enforced GLUT5 expression. (B) ${ }^{13}$ C-fructose concentration in A549 xenografts with or without enforced GLUT5 expression ( $n=5$ tumors for each group). (C) Tumor growth of A549 xenografts with or without ectopic GLUT5 expression. (D) Xenograft images and tumor weight of A549 cells with or without ectopic GLUT5 expression ( $n=10$ tumors for each group). Scale bar: $1 \mathrm{~cm}$. (E) Representative Western blot images exhibiting PCNA expression in A549 xenografts with or without enforced GLUT5 expression. Data shown as mean \pm SEM. ${ }^{* *} P<0.01 ;{ }^{* * *} P<0.001,2$-tailed Student's $t$ test.

\section{Discussion}

LC exhibits a hyperactive catabolic activity to satisfy its metabolic requirements (8). One of the most fundamental challenges is to determine those requisite nutrients used by LC cells in vivo to fuel metabolic activity. Apart from the well-known metabolic fuel glucose, lactate and branched-chain amino acids are considered alternative fuels for in vivo LC cells as demonstrated by recent studies $(40,41)$. In the current study, we demonstrate that circulating fructose is another crucial metabolic fuel for LC cells in vivo and that fructose utilization is strongly activated in LC tissues of patients under glucose-limited conditions. This study broadens our knowledge of the principal nutrients of LC cells in vivo.

It is an important issue to determine the source of fructose used by LC cells in patients and lung tumor xenografts in mice. Before surgery, patients with LC were provided a normal diet in the hospital, which usually contained desserts and fruits with plentiful fructose (27), such as sweet cakes, sweet cookies, kiwis, and apples. For the mice harboring lung tumor xenografts, they were fed a normal diet containing 10\% (weight/weight) sucrose (Supplemental Table 2). Sucrose is a disaccharide composed of 1 glucose and 1 fructose. Therefore, dietary fructose in the current study is an important source of fructose provision for LC cells in vivo. However, a limitation of this study is that fructose uptake by patients and mice was not quantitatively measured. Notably, a recent study found that the polyol pathway, which can transform glucose into fructose, is highly active in LC cells (42), thus yielding another potential source of fructose supply for LC cells in vivo.

One of the major findings of this study is that in vivo fructose utilization of LC cells is mediated mainly by GLUT5. In addition, GLUT5-mediated fructose utilization is indispensable for LC xenograft growth as demonstrated by 2 lines of evidence. First, $S L C 2 A 5$ deletion results in reduced fructose input and severely impaired lung tumor xenograft growth. However, tumor xenografts with $S L C 2 A 5$ ablation still slowly grew, indicating that other metabolic substrates, such as glutamine and lactate $(10,40)$, were 


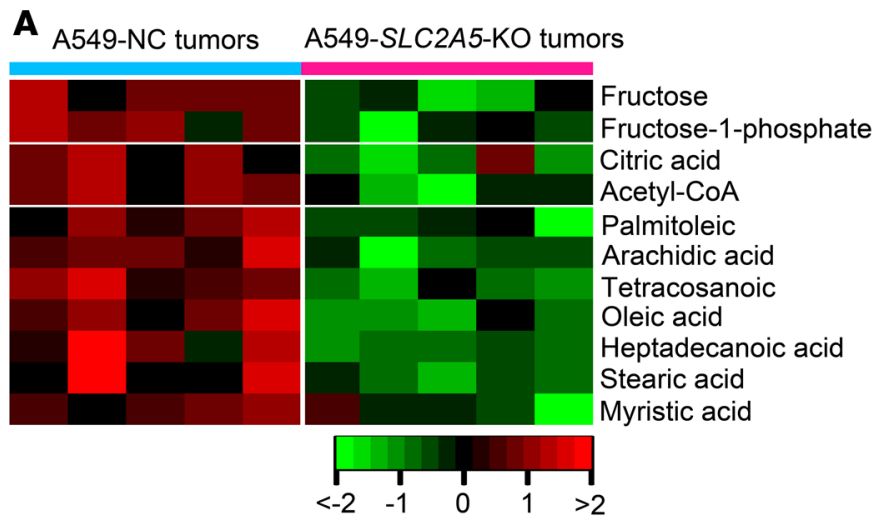

C

${ }^{13} \mathrm{C}$-fructose-derived metabolites in A549 xenografts
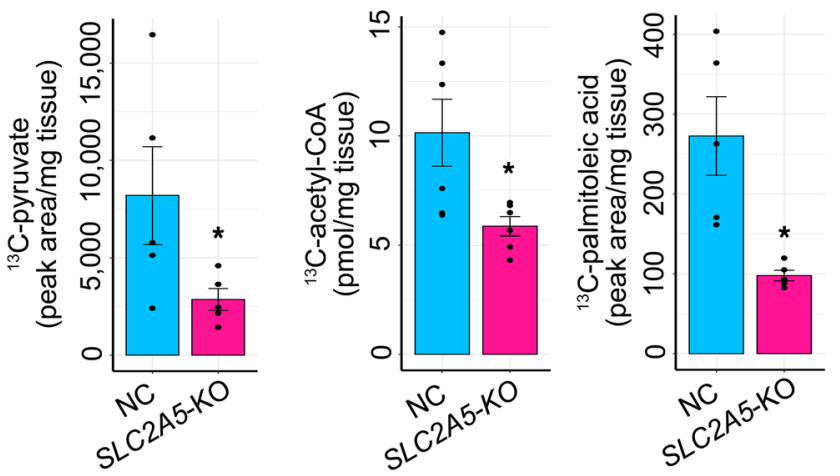

E

${ }^{13} \mathrm{C}$-fructose tracing in ex vivo lung slices of patients
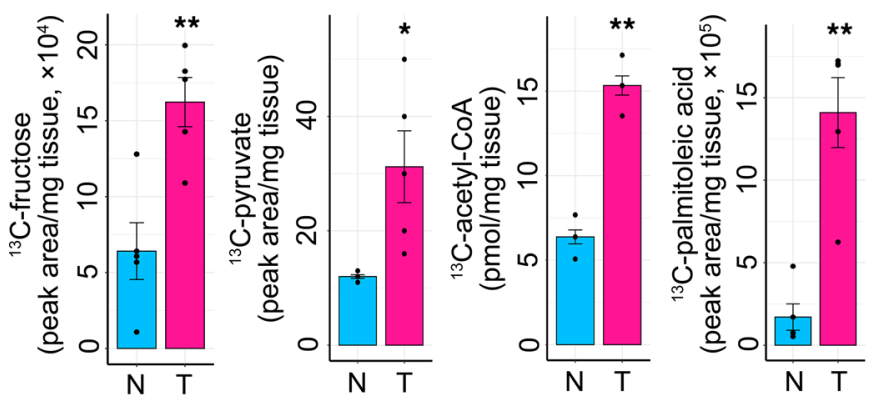

B A549-vector tumors A549-GLUT5 tumors

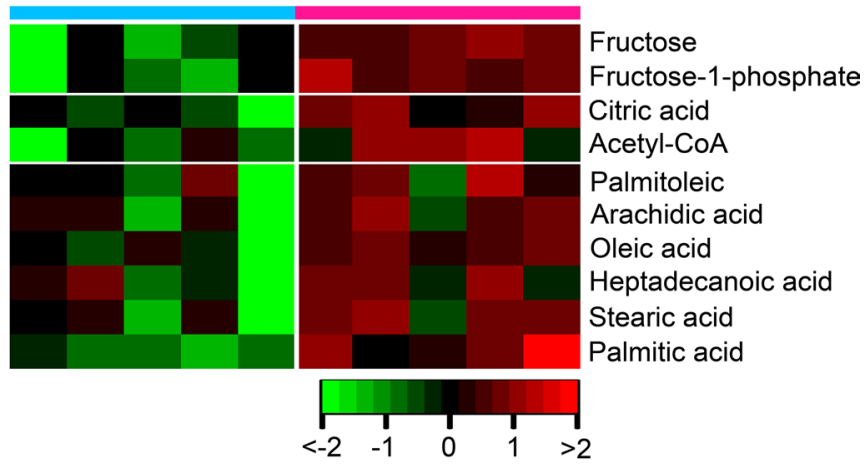

D

${ }^{13} \mathrm{C}$-fructose-derived metabolites in A549 xenografts
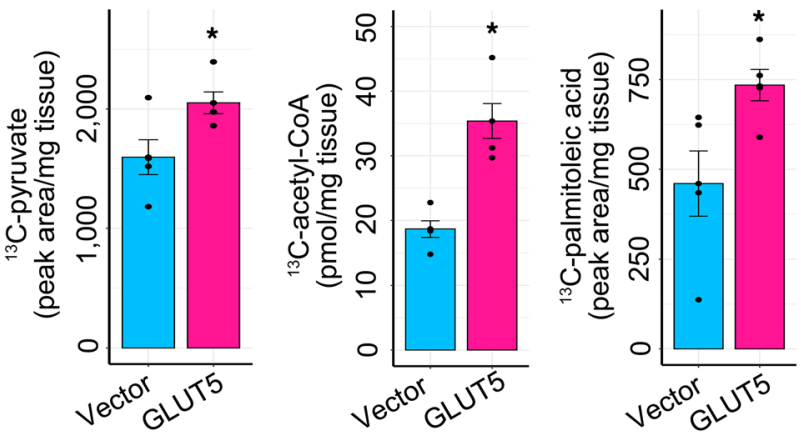

$\mathbf{F}$

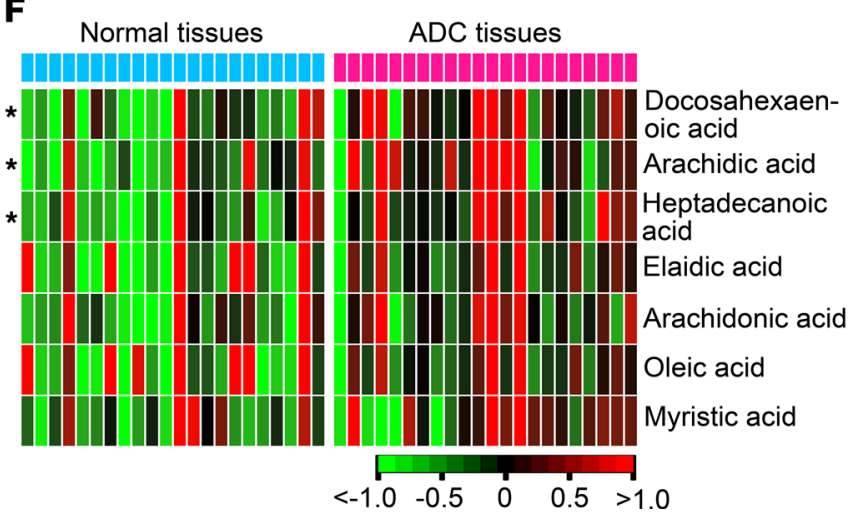

Figure 7. In vivo fructose utilization mediated by GLUT5 stimulates fatty acid synthesis and palmitoleic acid production. (A) Impact of impaired fructose utilization caused by SLC2A5 deletion on fructose input, fructose-derived metabolite generation, and synthesis of fatty acid precursors and fatty acids in A549 xenografts. (B) Influence of enhanced fructose utilization caused by ectopic GLUT5 expression on fructose input, fructosederived metabolite production, and synthesis of fatty acid precursors and fatty acids in $\mathrm{A} 549$ xenografts. (C) Production of ${ }^{13} \mathrm{C}$-metabolites derived from ${ }^{13} \mathrm{C}$-fructose in $\mathrm{A} 549$ xenografts with or without SLC2A5 ablation ( $n=5$ tumors for each group). (D) Generation of ${ }^{13} \mathrm{C}$-metabolites derived from ${ }^{13} \mathrm{C}$-fructose in $\mathrm{A} 549$ xenografts with or without enforced GLUT5 expression ( $n=5$ tumors for each group). (E) Tracer study in ex vivo lung tissues from LC patients with ADC. Fresh adjacent normal tissues $(n=5)$ and tumor tissues $(n=5)$ were enrolled for measurement of ${ }^{13} C$-fructose uptake and ${ }^{13} \mathrm{C}$-fructose-derived metabolite generation. (F) Analysis of fatty acids between adjacent normal and tumorous lung tissues of patients with lung ADC $(n=22)$. Data shown as mean \pm SEM. ${ }^{*} P<0.05 ;{ }^{* *} P<0.01$, 2-tailed Student's $t$ test.

potential alternative metabolic fuels for LC cells. Second, enforced expression of GLUT5 increased fructose uptake and expedited lung tumor xenograft growth. Of note, it is important to ascertain the upstream signals that stimulate GLUT5 expression in LC cells. Previous studies demonstrate that GLUT5 level can be modified by several metabolic diseases. Diabetes together with insulin can upregulate GLUT5 in muscle cells $(43,44)$, while obesity restrains GLUT5 expression in adipocytes and intestinal cells $(45,46)$. Nevertheless, the influence of metabolic diseases on GLUT5 expression of LC cells of patients needs to be explored. Moreover, ligand-activated nuclear hormone receptors, including liver X receptor $\alpha$, thyroid 
A

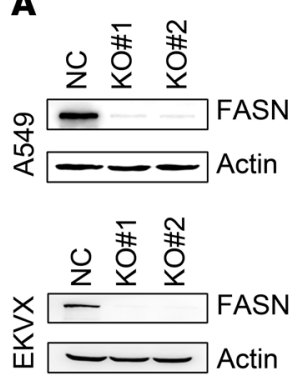

B

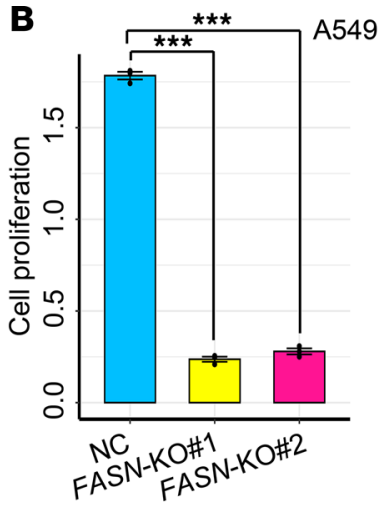

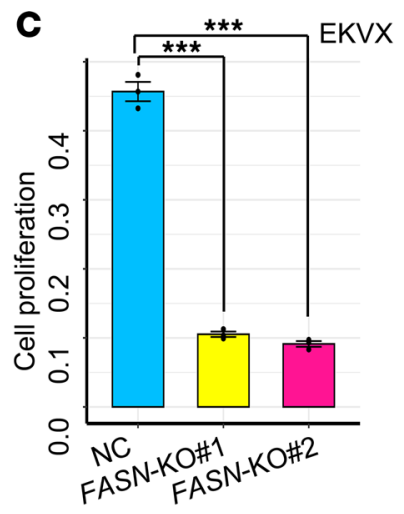

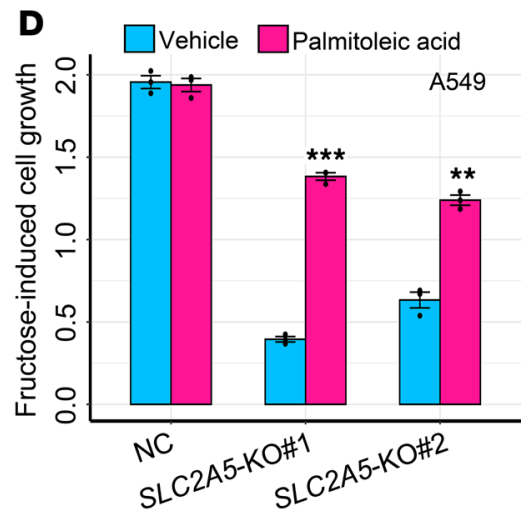

$\mathbf{E}$

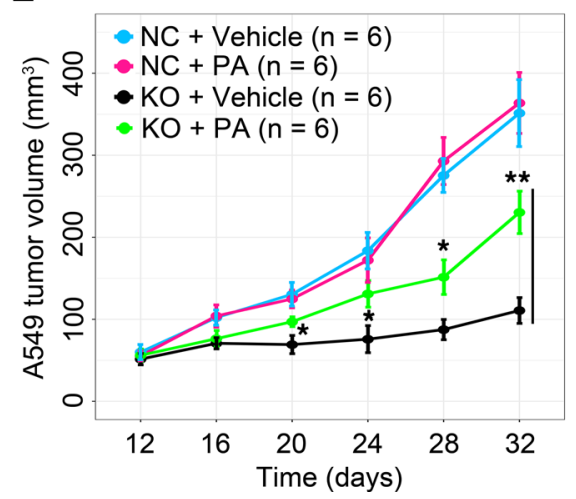

$\mathbf{F}$

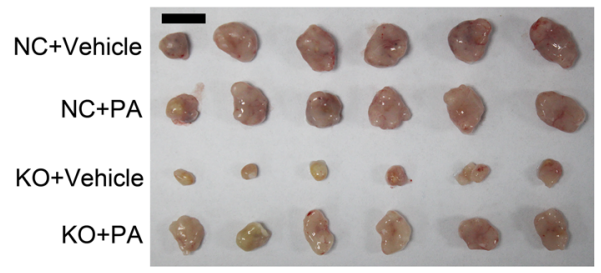

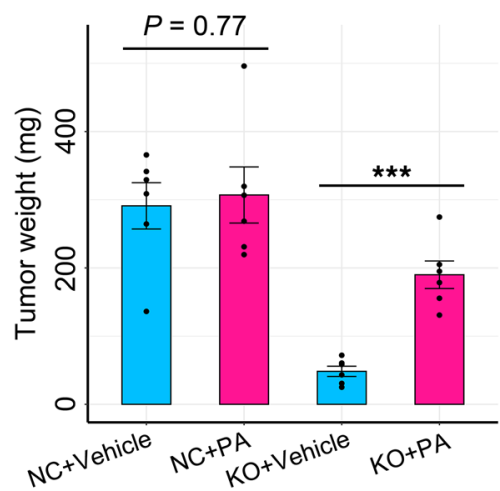

Figure 8. Downstream fatty acid synthesis from fructose is essential for LC growth. (A) Construction of A549 and EKVX cells with FASN knockout. (B and C) Impact of FASN ablation on fructose-induced cell growth of A549 and EKVX cells. Statistical analysis was conducted using 1-way ANOVA test. After conducting a homogeneity of variance test to confirm equal variance among subgroups, $P$ values were acquired from post hoc test using LSD algorism. Cumulative data are shown; $n=3$. (D) Palmitoleic acid supplement $(50 \mu \mathrm{M})$ restored the in vitro growth of A549 cells with SLC2A5 abrogation. Cumulative data are shown; $n=3$. (E) Restoration of A549 tumor growth with SLC2A5 knockout by palmitoleic acid (PA) administration. Mice bearing A549 tumors were divided into 4 groups and treated with vehicle or PA. (F) Tumor images and tumor weight of A549-NC cells with vehicle feeding ( $n=6$ ), A549-NC cells with PA feeding $(n=6)$, A549-SLC2A5-KO cells with vehicle feeding $(n=6)$, and A549-SLC2A5-KO cells with PA feeding $(n=6)$. Scale bar: $1 \mathrm{~cm}$. Data shown as mean \pm SEM. ${ }^{*} P<0.05 ;{ }^{* *} P<0.01 ;{ }^{* *} P<0.001$, 2-tailed Student's $t$ test.

hormone receptor $\alpha / \beta$, and retinoid X receptor $\alpha$, are able to strongly elevate GLUT5 expression at the transcriptional level in human cells (47). However, the impact of these receptors on GLUT5 expression of LC cells remains to be investigated. Under the condition of cancer, hypoxia is able to stimulate GLUT5 expression, and hypoxia-inducible factor $1 \alpha$ is positively correlated to GLUT5 in breast cancer (48). It is known that the tumor microenvironment of LC is hypoxic (49). Therefore, hypoxia and hypoxia-inducible factor $1 \alpha$ are potential upstream regulators of GLUT5 in LC.

Previous studies underscore the importance of fatty acid synthesis for LC growth $(50,51)$. In this study, we conducted metabolomic profiling coupled with isotope tracing of fructose and found that, under in vivo conditions, in vivo fructose metabolism mediated by GLUT5 was an essential pathway upstream of fatty acid synthesis, and this metabolic pathway was pivotal to retain endogenous fatty acid homeostasis. It is notable that fatty acids are reported to exert multifaceted roles in cancer. These metabolites not only serve as metabolic fuel but also function as second messengers for autocrine receptor signaling (52). Therefore, the tumor-promoting role of those fructose-derived fatty acids in LC is still to be further elucidated.

An intriguing finding of the current study is that an omega-7 monounsaturated fatty acid derived from fructose, palmitoleic acid, played a critical role in LC expansion in vitro and in vivo. We found that provision of exogenous palmitoleic acid was able to restore impaired LC growth caused by blockade of GLUT5-mediated fructose utilization. High level of serum palmitoleic acid is closely linked to increased cancer death (53), indicating an unfavorable role of this metabolite for cancer patients. However, the tumor-promoting role of this fatty acid in LC awaits further investigation. 
A

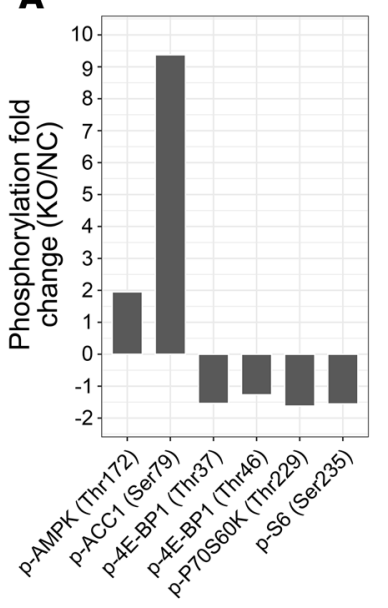

B

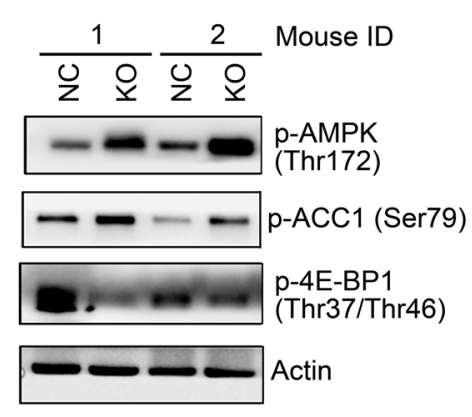

C

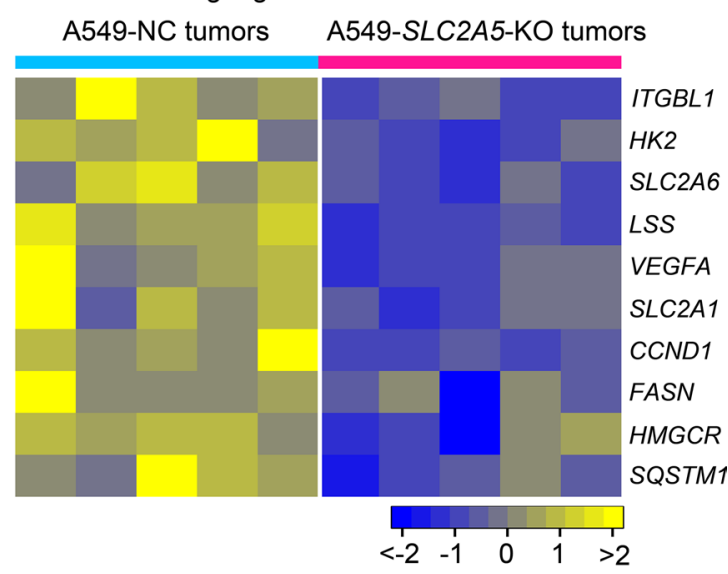

$\mathbf{F}$

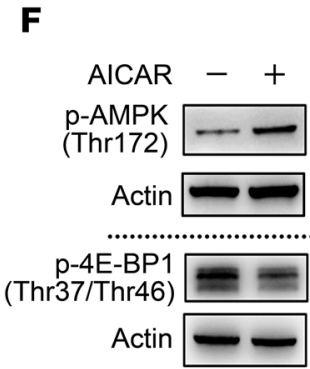

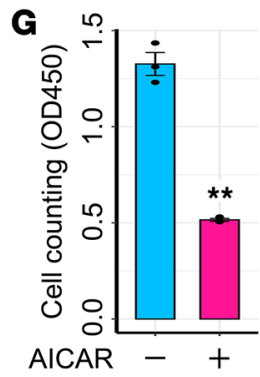

Figure 9. Fructose utilization mediated by GLUT5 influences the activity of AMPK/mTORC1 signaling in LC. (A) Phospho-protein profiling showing enhanced phosphorylation of AMPK and ACC1 and decreased phosphorylation of 4E-BP1, P70S6K, and S6 in A549 tumor xenograft with SLC2A5 deletion as relative to control A549 tumor xenograft. (B) Western blot approach validating the phosphorylation states of AMPK, ACC1, and 4E-BP1 between A549 tumor xenografts with SLC2A5 deletion and control A549 tumor xenografts. (C) RNA-Seq analysis showing repressed transcription of downstream target genes of mTORC1 signaling in A549 tumor xenografts with SLC2A5 abrogation. (D and E) Impact of fructose and glucose on AMPK (D) and 4E-BP1 (E) phosphorylation of A549 cells with or without SLC2A5 ablation. Control A549 cells and A549 cells with SLC2A5 deletion were starved in sugar-free medium for 2 hours and then were treated with $6 \mathrm{mM}$ fructose or $6 \mathrm{mM}$ glucose for 1 hour. Subsequently, cells were harvested for analysis. (F) The influence of an AMPK agonist, AICAR, on phosphorylation status of AMPK and 4E-BP1 in A549 cells cultured in fructose medium. Cells were treated with $1 \mathrm{mM}$ AICAR or vehicle for 6 hours. Samples from the same batch were run at different times. The corresponding loading controls were shown for each measurement. (G) The influence of AICAR (1 mM) on proliferation of A549 cells cultured in fructose medium. Cumulative data were shown; $n=3$. Data shown as mean \pm SEM. ${ }^{* *} P<0.01$, 2 -tailed Student's $t$ test.

Apart from fueling fatty acid synthesis in LC, GLUT5-mediated fructose utilization can also modulate the AMPK/mTORC1 signaling pathway to promote LC growth. Therefore, the present study demonstrates that enhanced fructose utilization expedited LC growth not only via rewiring downstream metabolism but also via activating an oncogenic signaling pathway. In addition, enhanced fructose utilization is closely associated with distal metastasis of LC and other cancers. Increased expression of GLUT5 is observed in LC liver metastasis (16). Intensified fructose metabolism promotes colon cancer metastasis toward the liver, and dietary fructose restriction restrains colon cancer liver metastasis (54). Taken together, a low-fructose diet should be tested in the future to determine whether it can ameliorate the neoplastic phenotypes of LC.

Because of the importance of in vivo fructose utilization mediated by GLUT5 for LC progression, this metabolic pathway is a potential therapeutic target. Indeed, we show that blocking fructose utilization by genetically deleting $S L C 2 A 5$ or by pharmacologically repressing GLUT5 could remarkably retard LC growth in vivo. Because there are no obvious side effects on mice with 2,5-AM administration, it is feasible to treat LC using this GLUT5 inhibitor.

\section{Methods}

Further information can be found in Supplemental Methods.

Cell lines, mouse strain, and antibodies. The human LC cell lines A549, EKVX, NCI-H226, and Hop-92 were obtained from National Cancer Institute, NIH, USA, while SK-MES-1 was purchased from Stem Cell Bank, Chinese Academy of Sciences. HEK293T cells (CRL-3216) were purchased from American 
A

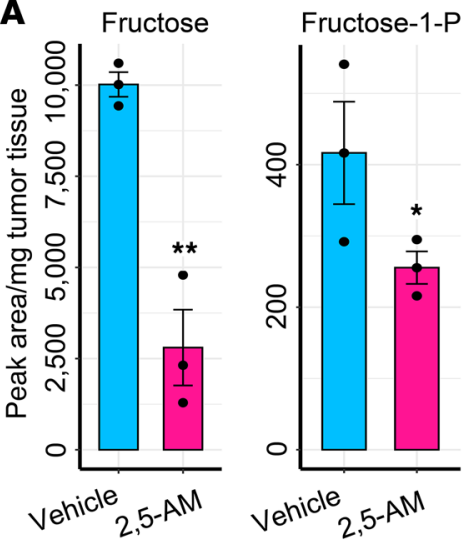

D

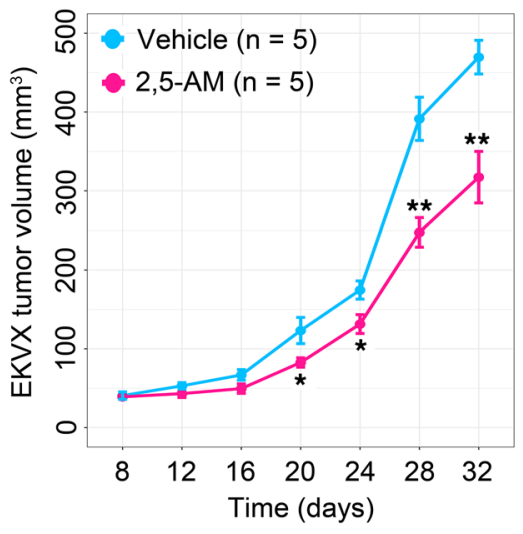

E

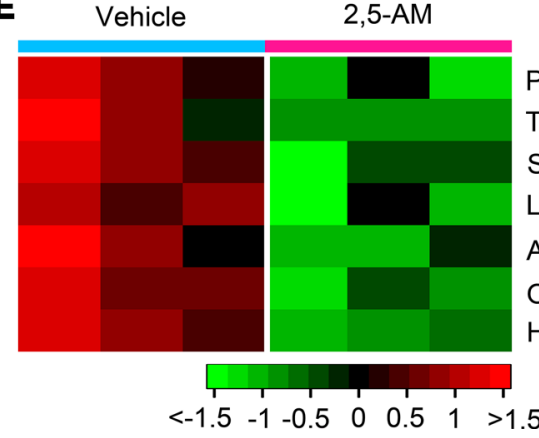

B

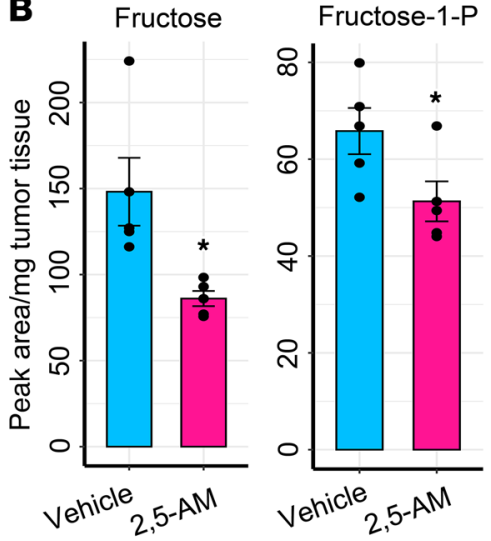

C

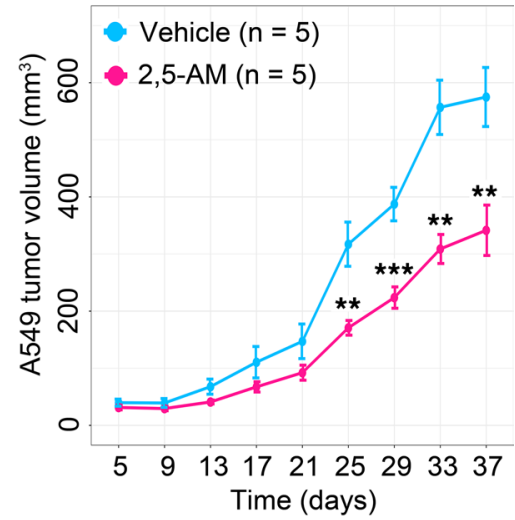

G

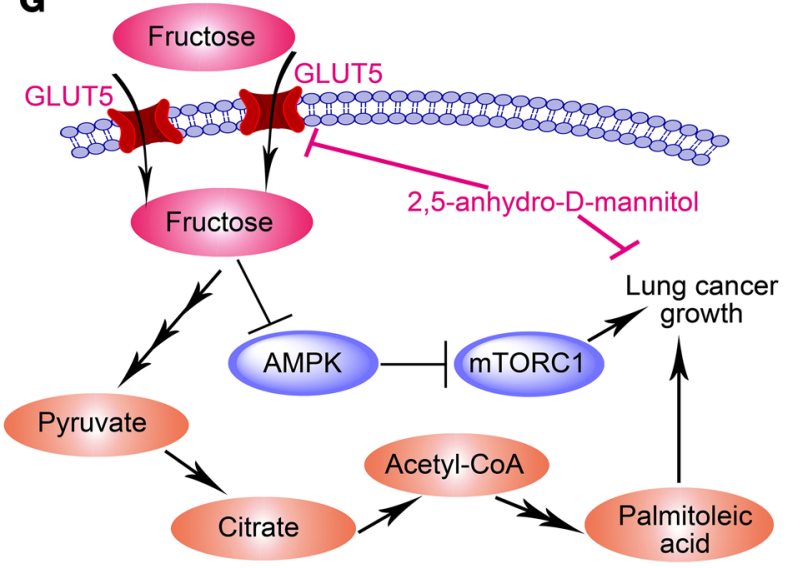

$\mathbf{F}$

Palmitoleic acid

Tetracosanic acid

Stearic acid

Linoleic acid

Arachidic acid

Oleic acid

Heptadecanoic acid

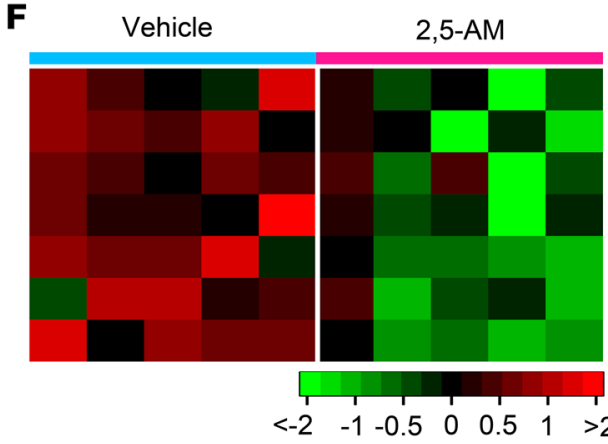

Palmitoleic acid

Tetracosanic acid

Stearic acid

Linoleic acid

Behenic acid

Caproic acid

Dodecanoic acid

Figure 10. In vivo pharmacological blockage of fructose utilization by 2,5-AM ameliorates the malignancy of LC. (A and B) 2,5-AM treatment inhibited fructose uptake and fructose-1-phosphate generation in A549 (A) ( $n=3$ tumors for each group) and EKVX (B) ( $n=5$ tumors for each group) xenografts. (C and D) Subcutaneous tumor growth of A549 (C) and EKVX (D) cells treated with vehicle or 2,5-AM. (E and F) Heatmap showing the alteration of fatty acids in A549 (E) ( $n=3$ tumors for each group) and EKVX (F) ( $n=5$ tumors for each group) xenografts treated with 2,5-AM. (C) A model depicting that in vivo fructose utilization mediated by GLUT5 promotes LC growth. On the one hand, incorporated fructose is used to synthesize downstream fatty acids. On the other hand, fructose utilization activates the oncogenic AMPK/mTORC1 signaling pathway. This gives rise to a therapeutic opportunity by blocking fructose utilization using a GLUT5 inhibitor, 2,5-AM. Data shown as mean \pm SEM. ${ }^{*} P<0.05$; ${ }^{* *} P<0.01$; ${ }^{* *} P<0.001$, 2-tailed Student's $t$ test.

Type Culture Collection. All cell lines were maintained in DMEM supplemented with 10\% fetal bovine serum (Thermo Fisher Scientific). We purchased 8-week-old female BALB/c-nude mice from Shanghai SLAC Laboratory Animal Co., Ltd. These mice were fed a normal diet containing 10\% sucrose (weight/ weight) (Supplemental Table 2). The primary antibodies involved in this study included anti-GLUT5 (Santa Cruz Biotechnology, sc-271055), anti-FASN (Cell Signaling Technology, 3180S), anti-PCNA (Cell Signaling Technology, 2856S), anti-Ki67 (Servicebio, GB13030-2), anti-cyclinD1 (Servicebio, GB13079), anti-cleaved caspase-3 (Servicebio, GB11532), anti-CD31 (Servicebio, GB13063), and anti-actin (CWBIO, CW0096M). Other key resources used in this study are summarized in Supplemental Tables 1 and 3. 
LC patients and samples. Patients with ADC or SCC were enrolled and provided a normal diet in Xinhua Hospital of Shanghai Jiaotong University School of Medicine. After fasting for 8 hours, these patients underwent surgery. Paired adjacent benign lung tissues and tumorous lung tissues were collected during the surgery. Tissue samples were immediately snap-frozen in liquid nitrogen and stored at $-80^{\circ} \mathrm{C}$. There were 2 batches of patient cohorts recruited in the current study. For the first batch of patient cohorts, they were enrolled in the past several months, and their tissue samples were used for expression analysis of genes, proteins, and metabolites. For the second batch of patient cohorts, they were enrolled in the past several years, and their tissue samples were used for tissue microarray preparation and IHC assay.

Metabolomic profiling of tissue samples. A combination of gas chromatography-time-of-flight mass spectrometry (GC-TOFMS, LECO Corp.) and ultra-performance liquid chromatography-tandem mass spectrometry (UPLCMS/MS, Waters Corp.) was used in the measurement of the small-molecule metabolites of tissue samples. Metabolomics assays were conducted by Metabo-Profile Inc. using previously published methods $(13,55)$. The metabolites were identified by comparison with the internal library built with the standard reference chemicals.

Fructose uptake analysis in the ex vivo study. This experiment was conducted using fresh slices of paired adjacent normal lung tissues and tumorous lung tissues resected from individual patients as described previously with minor modifications (10). Briefly, fresh lung tissue slices were immediately placed in 24-well plates containing glucose-free DMEM (Thermo Fisher Scientific) with 10\% dialyzed fetal bovine serum (dFBS, Thermo Fisher Scientific), $1.5 \mathrm{mM}$ fructose, and different concentrations of glucose, as indicated in Figure 1D, and then transferred to a $\mathrm{CO}_{2}$ incubator set at $37^{\circ} \mathrm{C}$ and $5 \% \mathrm{CO}_{2}$ for 72 hours. Spent media were collected to measure remaining fructose using a fructose colorimetric/fluorometric assay kit (MilliporeSigma) following the manufacturer's instructions. Fructose uptake was calculated by subtracting the remaining fructose from total fructose.

CRISPR/Cas 9 studies. We deleted SLC2A5 and FASN of LC cells using CRISPR/Cas9 technology as described (50). Briefly, each guide RNA (gRNA) duplex was inserted into the lenti-Guide-CRISPR-v2-puro vector, which was linearized by BsmBI endonuclease. The lentivirus was produced as follows: lenti-GuideCRISPR-v2-puro vector containing NC gRNA or lenti-Guide-CRISPR-v2-puro vector containing target gRNA was cotransfected with psPAX2 and pMD2.G plasmids into HEK293T cells using Lipofectamine 3000 (Thermo Fisher Scientific) following the manufacturer's instructions. Lentivirus-containing supernatants were collected and filtered $(0.45-\mu \mathrm{m}$ filter) to remove cells. Subsequently, LC cells were infected with the lentivirus harboring NC gRNA or lentivirus harboring target gRNA in the presence of $8 \mu \mathrm{g} / \mathrm{mL}$ polybrene. Transfected cells were selected with puromycin for 48 hours.

GLUT5 overexpression. We ectopically expressed GLUT5 in LC cells using an approach we described previously (39). LC cells were infected with control MigR1 retrovirus or MigR1 retrovirus with full-length human SLC2A5 cDNA. Transfected cells were sorted by using the selection marker green fluorescent protein.

Subcutaneous tumor studies and xenograft preparation for metabolomic profiling. A549-NC and A549-SLC2A5$\mathrm{KO}$, or EKVX-NC and EKVX-SLC2A5-KO, cells were subcutaneously injected into the left and right hind flanks of 8-week-old female BALB/c-nude mice, respectively. Similarly, A549-vector and A549-GLUT5 cells were subcutaneously infused into the left and right hind flanks of 8-week-old female BALB/c-nude mice, respectively. The tumor length and width were measured every 3 or 4 days using a caliper. Tumor sizes were calculated as $0.5 \times$ length $\times$ width $^{2}$. At the end of the experiments, tumor xenografts were resected for imaging and weighing. Subsequently, tumor xenografts were flash-frozen and stored in liquid nitrogen until metabolomic assay. Mouse studies were performed in specific pathogen-free facilities.

Tracer studies in mouse xenografts of LC cells and ex vivo lung tissue slices of LC patients. We performed ${ }^{13} \mathrm{C}-\mathrm{la}$ beled tracer studies using a previously reported protocol with a minor modification (10). For the tracer study in mouse xenografts, on the final day of the experiment, the tumor-bearing mice were injected via the tail vein with $100 \mu \mathrm{L} 1 \mathrm{M}\left[\mathrm{U}-{ }^{13} \mathrm{C} 6\right]$-D-fructose (Cambridge Isotope Laboratories) 3 times at 15 -minute intervals. One hour later, tumor xenografts were excised, weighed, and flash-frozen in liquid nitrogen. For the ex vivo tracer study, lung tissue slices of patients with LC were cultured in glucose-free DMEM with $10 \% \mathrm{dFBS}$ and $1.5 \mathrm{mM}\left[\mathrm{U}-{ }^{13} \mathrm{C} 6\right]$-D-fructose, and transferred to a $\mathrm{CO}_{2}$ incubator set at $37^{\circ} \mathrm{C}$ and $5 \% \mathrm{CO}_{2}$ for 72 hours. The tissue slices were then washed in PBS and frozen in liquid nitrogen. The metabolites of tissue samples were extracted and measured as previously described $(39,55)$.

${ }^{13} \mathrm{C}$-fructose and its derivatives, including ${ }^{13} \mathrm{C}$-pyruvate and ${ }^{13} \mathrm{C}$-palmitoleic acid, were analyzed using the GC-TOFMS platform and were monitored using ions at $\mathrm{m} / z 220,177$, and 132, respectively. ${ }^{13} \mathrm{C}$-Acetyl-CoA concentrations were quantitated by UPLC-MS/MS with 2 multiple reaction monitoring pairs (815/303 and 815/159 Da) as described previously (56). 
Palmitoleic acid rescue experiments. For the in vitro rescue study, palmitoleic acid was conjugated to bovine serum albumin (BSA) using a protocol described previously (50). Briefly, palmitoleic acid was solubilized in $100 \%$ alcohol at $50 \mathrm{mM}$ and combined with $4 \%$ fatty acid-free BSA (Equitech-Bio) in saline at a ratio of 1:4 to prepare a palmitoleic acid concentration of $10 \mathrm{mM}$. LC cells were cultured in glucose-free DMEM with 5\% dFBS and $6 \mathrm{mM}$ fructose. Palmitoleic acid-BSA was added into culture medium at a final concentration of $50 \mu \mathrm{M}$.

For the in vivo rescue study, palmitoleic acid was dissolved in PBS. After 10 days of subcutaneous injection of A549-NC or A549-SLC2A5-KO cells, palmitoleic acid solution was given to the tumor-bearing mice by oral administration every other day with a dosage of $1 \mathrm{mg} /$ mouse. PBS with the same volume was given to the mice of the vehicle group. Notably, palmitoleic acid solution was mixed thoroughly before each oral administration.

Phospho-protein profiling by phospho-antibody array. Phospho-protein profiling was conducted as previously illustrated (57). Xenograft tumor tissue lysates were applied to the Phospho Explorer Antibody Array (PEX100, Full Moon Biosystems Inc.) following the manufacturer's protocol. The phosphorylation ratio of each antibody (phosphorylated and matching unphosphorylated values were denoted by "phospho" and "unphospho" in both the control data and experiment data) was computed using an equation as previously described (58).

RNA-Seq and data analysis. RNA-Seq was performed by Cloudseq Biotech Inc. following the published procedure (59). Paired-end reads were harvested from Illumina HiSeq 4000 sequencer and were quality controlled by Q30. After 3' adapter trimming and low-quality read removal by cutadapt software (v1.9.3), the high-quality, clean reads were aligned to the reference genome (UCSC hg19) with hisat2 software (v2.0.4). Then, guided by the Ensembl gtf gene annotation file, cuffdiff software (part of cufflinks) was used to get fragments per kilobase of transcript per million mapped reads values as the expression profiles of mRNA. Student's 2-tailed $t$ test was used to identify differentially expressed genes downstream of mTORC1 signaling. RNA-Seq raw data were deposited to the public database ArrayExpress with accession number E-MTAB-8572 (http://www.ebi.ac.uk/arrayexpress).

2,5-AM treatment of mice with lung tumor xenografts. We implemented 2,5-AM administration to the lung tumor-bearing mice as previously described (39). The reagent was dissolved in physiological saline and intraperitoneally injected into lung tumor-bearing mice at a dose of $150 \mathrm{mg} / \mathrm{kg} / \mathrm{d}$ at day 10 after subcutaneous transplantation of lung cells until the mice were killed. The same volume of physiological saline was given to the vehicle group.

Statistics. Statistical analysis was performed using R software (R version 3.4.3, http://www.r-project.org). Significant differences between 2 groups were computed using Wilcoxon's rank-sum test for data with skewed distribution or Student's $t$ test for data with normal distribution. Notably, Wilcoxon's rank-sum test and Student's $t$ test were 2 tailed. One-way analysis of variance (ANOVA) was carried out for comparison of variance of the variable among 3 groups. Post hoc tests for multiple comparisons were conducted using LSD method with equal variances or Dunnett's T3 algorithm with unequal variances. Survival analysis was conducted by means of the Kaplan-Meier method followed by log-rank test. The association between 2 variables was calculated using Spearman's rank correlation method. A $P$ value less than 0.05 was considered significant.

Study approval. All participants provided informed written consent in accordance with the regulation of the Institutional Review Boards of Xinhua Hospitals in agreement with the Declaration of Helsinki. All animal protocols and experiments were approved by the Institutional Animal Care and Use Committee of Fudan University: SYXK(Hu)2014-0029.

\section{Author contributions}

WLC and LJ conceptualized the study. MW, LM, RB, LW, XX, GY, LL, and QC provided human LC samples. WLC, XJ, and DL were responsible for metabolomics and metabolic tracer assays. WLC, XJ, QL, $\mathrm{DL}, \mathrm{HT}$, and LC were responsible for in vitro and ex vivo assays. XJ, QL, DL, CD, and WLC conducted the mouse study. WLC, XJ, and QL were responsible for bioinformatics and statistical analysis. WLC and LJ wrote the original draft of the manuscript. WLC, LJ, WJ, and WW edited the final draft.

\section{Acknowledgments}

This work was supported by the National Key R\&D Program of China (2016YFA0501800), National Thirteenth Five-Year Science and Technology Major Special Project for New Drug Innovation and Development (2017ZX09304001), National Scientific and Technological Major Special Project of China (2018ZX09201008002), National Natural Science Foundation of China (81770147, 31970708, 81625018, 81572340, 81802891), 
Research Fund of Shanghai Municipal Commission of Health (20174Y0090), Shanghai Rising-Star Program (18QA1404100), Program for Professor of Special Appointment (Eastern Scholar) at Shanghai Institutions of Higher Learning, Shanghai Youth Talent Program, Program of Shanghai Academic/Technology Research Leader (18XD1403800), Shanghai Chenguang Program (18CG47), Gaofeng Clinical Medicine Grant of Shanghai Municipal Education Commission, and Xinling Scholar Program of Shanghai University of Traditional Chinese Medicine.

Address correspondence to: Wen-Lian Chen, Cancer Institute, Longhua Hospital Shanghai University of Traditional Chinese Medicine, 725 South Wanping Road, Building 12, Room 607, Shanghai 200032, China. Phone: 86.21.64385700 ext. 9602; Email: chenw18412@shutcm.edu.cn. Or to: Lijun Jia, Cancer Institute, Longhua Hospital Shanghai University of Traditional Chinese Medicine, 725 South Wanping Road, Building 12, Room 601, Shanghai 200032, China. Phone: 86.21.64385700 ext. 9601; Email: 1jjia@shutcm.edu.cn.

1. Hirsch FR, et al. Lung cancer: current therapies and new targeted treatments. Lancet. 2017;389(10066):299-311.

2. Chen Z, Fillmore CM, Hammerman PS, Kim CF, Wong KK. Non-small-cell lung cancers: a heterogeneous set of diseases. Nat Rev Cancer. 2014;14(8):535-546.

3. Inamura K. Lung cancer: understanding its molecular pathology and the 2015 WHO classification. Front Oncol. $2017 ; 7: 193$.

4. Saito M, Shiraishi K, Kunitoh H, Takenoshita S, Yokota J, Kohno T. Gene aberrations for precision medicine against lung adenocarcinoma. Cancer Sci. 2016;107(6):713-720.

5. Qin A, Coffey DG, Warren EH, Ramnath N. Mechanisms of immune evasion and current status of checkpoint inhibitors in non-small cell lung cancer. Cancer Med. 2016;5(9):2567-2578.

6. Vander Heiden MG, DeBerardinis RJ. Understanding the intersections between metabolism and cancer biology. Cell. 2017;168(4):657-669.

7. Halbrook CJ, Lyssiotis CA. Employing metabolism to improve the diagnosis and treatment of pancreatic cancer. Cancer Cell. 2017;31(1):5-19.

8. Fan TW, et al. Altered regulation of metabolic pathways in human lung cancer discerned by (13)C stable isotope-resolved metabolomics (SIRM). Mol Cancer. 2009;8:41.

9. Hori S, et al. A metabolomic approach to lung cancer. Lung Cancer. 2011;74(2):284-292.

10. Sellers K, et al. Pyruvate carboxylase is critical for non-small-cell lung cancer proliferation. J Clin Invest. 2015;125(2):687-698.

11. Vander Heiden MG, Cantley LC, Thompson CB. Understanding the Warburg effect: the metabolic requirements of cell proliferation. Science. 2009;324(5930):1029-1033.

12. Zhao Y, Butler EB, Tan M. Targeting cellular metabolism to improve cancer therapeutics. Cell Death Dis. $2013 ; 4:$ e532.

13. Chen WL, et al. A distinct glucose metabolism signature of acute myeloid leukemia with prognostic value. Blood. 2014;124(10):1645-1654.

14. Feinberg T, et al. Cancerous glucose metabolism in lung cancer-evidence from exhaled breath analysis. J Breath Res. 2016;10(2):026012.

15. Younes M, Brown RW, Stephenson M, Gondo M, Cagle PT. Overexpression of Glut1 and Glut3 in stage I nonsmall cell lung carcinoma is associated with poor survival. Cancer. 1997;80(6):1046-1051.

16. Kurata T, Oguri T, Isobe T, Ishioka S, Yamakido M. Differential expression of facilitative glucose transporter (GLUT) genes in primary lung cancers and their liver metastases. Jpn J Cancer Res. 1999;90(11):1238-1243.

17. Zaizen Y, et al. Prognostic significance of total lesion glycolysis in patients with advanced non-small cell lung cancer receiving chemotherapy. Eur J Radiol. 2012;81(12):4179-4184.

18. Xie H, et al. Targeting lactate dehydrogenase--a inhibits tumorigenesis and tumor progression in mouse models of lung cancer and impacts tumor-initiating cells. Cell Metab. 2014;19(5):795-809.

19. Hensley CT, et al. Metabolic heterogeneity in human lung tumors. Cell. 2016;164(4):681-694.

20. Douard V, Ferraris RP. The role of fructose transporters in diseases linked to excessive fructose intake. J Physiol (Lond). 2013;591(2):401-414.

21. Barone S, et al. Slc2a5 (Glut5) is essential for the absorption of fructose in the intestine and generation of fructose-induced hypertension. J Biol Chem. 2009;284(8):5056-5066.

22. Hirabayashi J. On the origin of elementary hexoses. Q Rev Biol. 1996;71(3):365-380.

23. Bollen M, Keppens S, Stalmans W. Specific features of glycogen metabolism in the liver. Biochem J. 1998;336(pt 1):19-31.

24. Macdonald IA. Carbohydrate as a nutrient in adults: range of acceptable intakes. Eur J Clin Nutr. 1999;53(suppl 1):S101-S106.

25. Hannou SA, Haslam DE, McKeown NM, Herman MA. Fructose metabolism and metabolic disease. J Clin Invest. 2018;128(2):545-555.

26. Charrez B, Qiao L, Hebbard L. The role of fructose in metabolism and cancer. Horm Mol Biol Clin Investig. 2015;22(2):79-89.

27. Port AM, Ruth MR, Istfan NW. Fructose consumption and cancer: is there a connection? Curr Opin Endocrinol Diabetes Obes. 2012;19(5):367-374.

28. Li X, et al. A splicing switch from ketohexokinase-C to ketohexokinase-A drives hepatocellular carcinoma formation. Nat Cell Biol. 2016;18(5):561-571.

29. Weng Y, et al. SLC2A5 promotes lung adenocarcinoma cell growth and metastasis by enhancing fructose utilization. Cell Death Discov. 2018;4:38.

30. Godoy A, et al. Differential subcellular distribution of glucose transporters GLUT1-6 and GLUT9 in human cancer: ultrastructural localization of GLUT1 and GLUT5 in breast tumor tissues. J Cell Physiol. 2006;207(3):614-627. 
31. Hirahatake KM, Meissen JK, Fiehn O, Adams SH. Comparative effects of fructose and glucose on lipogenic gene expression and intermediary metabolism in HepG2 liver cells. PLoS ONE. 2011;6(11):e26583

32. Ikediobi ON, et al. Mutation analysis of 24 known cancer genes in the NCI-60 cell line set. Mol Cancer Ther. 2006;5(11):2606-2612.

33. Carroll PA, et al. Deregulated Myc requires MondoA/Mlx for metabolic reprogramming and tumorigenesis. Cancer Cell. 2015;27(2):271-285.

34. Zhang CS, et al. Fructose-1,6-bisphosphate and aldolase mediate glucose sensing by AMPK. Nature. 2017;548(7665):112-116.

35. Herzig S, Shaw RJ. AMPK: guardian of metabolism and mitochondrial homeostasis. Nat Rev Mol Cell Biol. 2018;19(2):121-135.

36. Fumarola C, Bonelli MA, Petronini PG, Alfieri RR. Targeting PI3K/AKT/mTOR pathway in non small cell lung cancer. Biochem Pharmacol. 2014;90(3):197-207.

37. Durán RV, et al. Glutaminolysis activates Rag-mTORC1 signaling. Mol Cell. 2012;47(3):349-358.

38. Laplante M, Sabatini DM. Regulation of mTORC1 and its impact on gene expression at a glance. J Cell Sci. 2013;126(pt 8):1713-1719.

39. Chen WL, et al. Enhanced fructose utilization mediated by SLC2A5 is a unique metabolic feature of acute myeloid leukemia with therapeutic potential. Cancer Cell. 2016;30(5):779-791.

40. Faubert B, et al. Lactate metabolism in human lung tumors. Cell. 2017;171(2):358-371.e9.

41. Mayers JR, et al. Tissue of origin dictates branched-chain amino acid metabolism in mutant Kras-driven cancers. Science. 2016;353(6304):1161-1165.

42. Schwab A, et al. Polyol pathway links glucose metabolism to the aggressiveness of cancer cells. Cancer Res. 2018;78(7):1604-1618.

43. Stuart CA, Howell ME, Yin D. Overexpression of GLUT5 in diabetic muscle is reversed by pioglitazone. Diabetes Care. 2007;30(4):925-931.

44. Hajduch E, Litherland GJ, Turban S, Brot-Laroche E, Hundal HS. Insulin regulates the expression of the GLUT5 transporter in L6 skeletal muscle cells. FEBS Lett. 2003;549(1-3):77-82.

45. Litherland GJ, Hajduch E, Gould GW, Hundal HS. Fructose transport and metabolism in adipose tissue of Zucker rats: diminished GLUT5 activity during obesity and insulin resistance. Mol Cell Biochem. 2004;261(1-2):23-33.

46. Deal RA, Tang Y, Fletcher R, Torquati A, Omotosho P. Understanding intestinal glucose transporter expression in obese compared to non-obese subjects. Surg Endosc. 2018;32(4):1755-1761.

47. Zwarts I, et al. Identification of the fructose transporter GLUT5 (SLC2A5) as a novel target of nuclear receptor LXR. Sci Rep. 2019;9(1):9299.

48. Hamann I, et al. Expression and function of hexose transporters GLUT1, GLUT2, and GLUT5 in breast cancer-effects of hypoxia. FASEB J. 2018;32(9):5104-5118.

49. Salem A, et al. Targeting hypoxia to improve non-small cell lung cancer outcome. J Natl Cancer Inst. 2018;110(1).

50. Svensson RU, et al. Inhibition of acetyl-CoA carboxylase suppresses fatty acid synthesis and tumor growth of non-small-cell lung cancer in preclinical models. Nat Med. 2016;22(10):1108-1119.

51. Merino Salvador M, et al. Lipid metabolism and lung cancer. Crit Rev Oncol Hematol. 2017;112:31-40.

52. Röhrig F, Schulze A. The multifaceted roles of fatty acid synthesis in cancer. Nat Rev Cancer. 2016;16(11):732-749.

53. Byberg L, Kilander L, Warensjö Lemming E, Michaëlsson K, Vessby B. Cancer death is related to high palmitoleic acid in serum and to polymorphisms in the SCD-1 gene in healthy Swedish men. Am J Clin Nutr. 2014;99(3):551-558.

54. Bu P, et al. Aldolase B-mediated fructose metabolism drives metabolic reprogramming of colon cancer liver metastasis. Cell Metab. 2018;27(6):1249-1262.e4.

55. Qiu Y, et al. A distinct metabolic signature of human colorectal cancer with prognostic potential. Clin Cancer Res. 2014;20(8):2136-2146.

56. Cai L, Sutter BM, Li B, Tu BP. Acetyl-CoA induces cell growth and proliferation by promoting the acetylation of histones at growth genes. Mol Cell. 2011;42(4):426-437.

57. Jia D, et al. Amplification of MPZL1/PZR promotes tumor cell migration through Src-mediated phosphorylation of cortactin in hepatocellular carcinoma. Cell Res. 2014;24(2):204-217.

58. Kang S, et al. p90 ribosomal S6 kinase 2 promotes invasion and metastasis of human head and neck squamous cell carcinoma cells. J Clin Invest. 2010;120(4):1165-1177.

59. Du Y, et al. SUMOylation of the m6A-RNA methyltransferase METTL3 modulates its function. Nucleic Acids Res 2018;46(10):5195-5208. 